\title{
Effect of Self-Assembly of Oxalamide Based Organic Compounds on Melt Behavior, Nucleation, and Crystallization of Isotactic Polypropylene
}

\author{
Carolus H. R. M. Wilsens, ${ }^{*}{ }^{\dagger}$ Laurence G. D. Hawke, ${ }^{\dagger}$ Enrico M. Troisi, ${ }^{\ddagger}$ Daniel Hermida-Merino,
} Gijs de Kort, ${ }^{\dagger}$ Nils Leoné, ${ }^{\dagger}$ Ketie Saralidze, ${ }^{\dagger}$ Gerrit W. M. Peters, ${ }^{\ddagger}$ and Sanjay Rastogi ${ }^{\dagger}$

${ }^{\dagger}$ Aachen-Maastricht Institute of BioBased Materials (AMIBM), Maastricht University, P.O. Box 616, 6200MD, Maastricht, The Netherlands

${ }^{\ddagger}$ Department of Mechanical Engineering, Materials Technology Institute, Eindhoven University of Technology, P.O. Box 513, 5600 $\mathrm{MB}$, Eindhoven, The Netherlands

${ }^{\S}$ Netherlands Organisation for Scientific Research (NWO), DUBBLE@ESRF BP CS40220, 38043 Grenoble, France

Supporting Information

ABSTRACT: We report on the effect of an aliphatic oxalamide based nucleating agent $($ OXA3,6) on the melt and crystallization behavior of isotactic polypropylene (iPP) under defined shear conditions. Through polarized optical microscopy, we demonstrate that OXA3,6 self-assembles from the $\mathrm{PP}$ melt into rhombic crystals whereas their size and distribution proved highly dependent on the employed cooling rates. The presence of 0.5 wt \% of OXA3,6 in iPP results in a significant suppression in $\mathrm{PP}$ melt viscosity, which could not be explained via molecular modeling. A possible cause for the drop in viscosity in the presence of OXA3,6 is attributed to the interaction (absorption) of high molecular weight $i \mathrm{PP}$ chains with the nucleating agent, thereby suppressing their contribution to the viscoelastic response of the melt. This proposed mechanism for the suppression in melt viscosity appears similar to that encountered by the homogeneous distribution of nanoparticles such as CNTs, graphene, and silica. Shear experiments, performed using a slit flow device combined with smallangle X-ray diffraction measurements, indicate that crystallization is significantly enhanced in the presence of OXA3,6 at relatively low shear rates despite its lowered sensitivity to shear. This enhancement in crystallization is attributed to the shear alignment of the rhombic OXA3,6 crystals that provide surface for $i \mathrm{PP}$ kebab growth upon cooling. Overall, the suppression in melt viscosity in combination with enhanced nucleation efficiency at low as well as high shear rates makes this self-assembling oxalamide based nucleating agent a promising candidate for fast processing.

\section{INTRODUCTION}

Hydrogen-bonding amide based organic components have attracted significant attention in the recent years due to their efficient role as nucleating agent (NA). The one- or two-step synthesis route allows for an easy variation in chemical structure and thereby provides a toolbox to tailor and optimize their nucleating efficiency for various polymers. Examples of such nucleating agents include amide, ${ }^{1-3}$ oxalamide, ${ }^{4-8}$ and hydrazide 9 based organic compounds. The organic nature of these compounds allows their miscibility in the polymer melt and self-assembly in the polymer matrix upon cooling. Subsequently, these crystallized NA aggregates provide surface for nucleation of the polymer and initiate crystallization. Over the years, through molecular variation of the amide motifs, numerous examples of hydrogen-bonding organic NAs for various polymers including polypropylene, poly(lactic acid), poly(hydroxyalkanoates), and poly(butylene succinate), among others, ${ }^{1-12}$ are reported.
In contrast to sorbitol type of organic nucleating agents, that form one-dimensional nanofibrillar networks, the self-assembly of the oxalamide based NAs results in three-dimensional NA crystals. ${ }^{13,14}$ Since the available surface for nucleation strongly influences the overall nucleating efficiency, a finely dispersed one-dimensional nanofibrillar network in a polymer melt is preferred over two- or three-dimensional NA crystals. In previous work, ${ }^{4}$ we have demonstrated that the crystal morphology and surface-to-volume ratio of oxalamide based nucleating agents can be improved significantly upon the application of shear at elevated temperatures. In fact, the nucleating efficiency observed under those conditions, indicated by the onset of crystallization, increases significantly and becomes comparable to that of the organic sorbitol NAs.

Received: March 7, 2018

Revised: May 22, 2018

Published: June 21, 2018 
It is well-known that crystallization of polymers can be enhanced significantly by the application of shear. Shear flow orients the chains molecularly resulting in the formation of bundles of oriented chains, also known as point-like nuclei. These point-like nuclei are presumed to aggregate and align along the flow direction forming row nuclei that develop further into a shish through infilled growth. ${ }^{15,16}$ In turn, the shish suppresses the nucleation barrier and facilitates crystal growth perpendicular to the shish and shear direction. The lifetime of such shear-induced precursors or row nuclei decreases with decreasing shear intensity, molecular weight, and/or undercooling as these shear-induced precursors can relax back to point-like precursors or even fully relax after sufficient holding time. ${ }^{17,18}$ Upon the addition of (nano)fillers the effect of shear flow on crystallization becomes rather complex. First, these fillers can provide a surface for heterogeneous nucleation. Second, in proximity of particles the local velocity gradient can increase considerably, synergistically enhancing the crystallization kinetics. Third, physical adsorption of the polymer on the surface of the filler can occur, thereby affecting the rheological and thus the shear response of the material. ${ }^{20}$

Though the underlying mechanism is not known, the synergistic effect of shear on the crystallization of $i \mathrm{PP}$ containing oxalamide based nucleating agents seems promising for application under processing conditions where high shear and high cooling rates are required. To obtain more insight into this synergistic crystallization behavior, in this study we assess the performance of nucleating agent OXA3,6 (Scheme 1) under various shear conditions and evaluate its effect on the

Scheme 1. Chemical Structure of Nucleating Agent OXA3,6

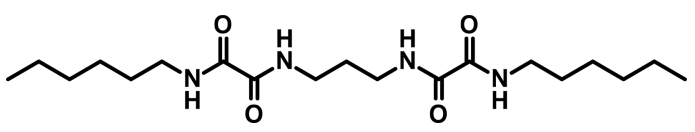

crystallization behavior of $i \mathrm{PP}$ upon cooling. The employed multipass rheometry (MPR) setup equipped with diamond windows allows us to subject the polymer melt to high shear rates in slit-flow geometry and to follow the structure development at different length scales using the synchrotron radiation facilities available on beamline BM26 at the ESRF. In other words, this setup provides a unique possibility of investigating the crystallization behavior of this polymer under controlled but high shear rates.

\section{EXPERIMENTAL SECTION}

Material Preparation and Blending Process. The developed compounds were melt-blended with linear $i \mathrm{PP}$ from Borealis, obtained directly in the powder form from the reactor (T1410 grade). Antioxidant Irganox 1010 (1 wt \%) was added to all samples to limit degradation during processing. The nucleating agent OXA3,6 has been synthesized as described in a previous publication. ${ }^{4}$ All materials were dried in vacuo at $80{ }^{\circ} \mathrm{C}$ overnight, prior to processing. Extrusion and blending were performed at $220{ }^{\circ} \mathrm{C}$ for $3 \mathrm{~min}$ at 100 $\mathrm{rpm}$ in a single-screw extruder. The extrudate was quenched in water and cut into pellets. The pellets were compression molded after drying overnight in vacuo and cut into MPR bars of $1.5 \times 6 \times 120$ $\mathrm{mm}^{3}$.

Shear and Crystallization Protocols Using Multipass Rheometry. Slit-flow experiments were performed using a multipass rheometer (MPR). ${ }^{21}$ A polymer bar of $1.5 \times 6 \times 120 \mathrm{~mm}^{3}$ is confined between two pistons that are hydraulically driven. Pressurization can be imposed on the sample by moving the pistons toward each other. Similarly, slit-flow experiments can be performed by moving the pistons in the same direction. The pressure in the polymer sample can be carefully controlled up to 1000 bar by two pressure transducers located close to the two pistons. Moreover, the two pressure transducers allow for measuring the pressure difference over the slit. The sample is cooled by pumping a cooling medium through the cell. Two diamond windows having a $60^{\circ}$ opening located in middle of the slit, allowing us to monitor the structure development of the samples using X-ray diffraction techniques.

Experiments using MPR were performed in two stages. First, the samples were loaded in the MPR and heated to $200{ }^{\circ} \mathrm{C}$. After reaching the set temperature of $200{ }^{\circ} \mathrm{C}$ the samples were pressured and kept isobaric at $50 \mathrm{bar}$, and an isotherm of $5 \mathrm{~min}$ was applied to ensure that all nucleating agent has molten and the thermal history of the sample is eradicated. In the following step, the sample was cooled to the desired temperature at a rate of $10{ }^{\circ} \mathrm{C} / \mathrm{min}$. Once the desired temperature was reached $\left(170{ }^{\circ} \mathrm{C}\right)$, a shear pulse was given after subjecting the sample to another isotherm of $5 \mathrm{~min}$. Taking into account the limitations of the maximum movement of the pistons, the piston displacement was fixed to $15 \mathrm{~mm}$. The piston velocity was varied from 1 to $100 \mathrm{~mm} / \mathrm{s}$ (translating to apparent wall shear rates of 4-400 $\mathrm{s}^{-1}$ ). In the second stage of the experiment, for the crystallization studies, immediately after the shear pulse, the sample was actively cooled using a thermostat containing oil at $100{ }^{\circ} \mathrm{C}$. For samples without shear pulse, the cooling was triggered immediately
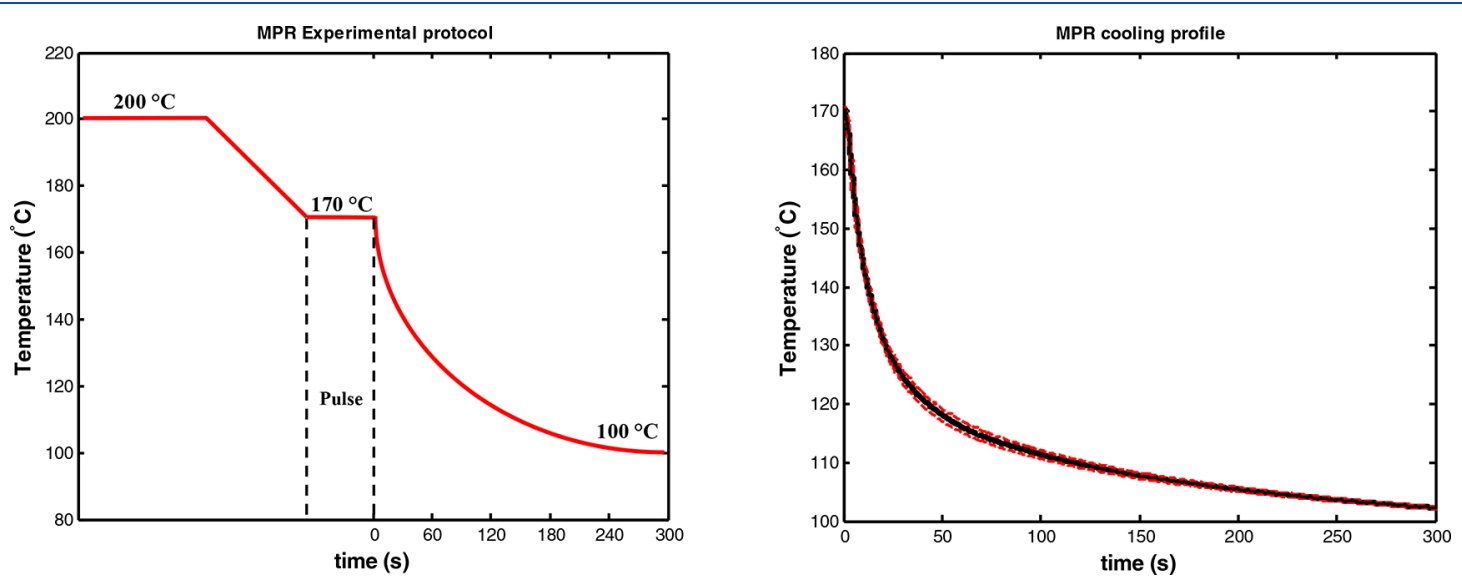

Figure 1. Experimental protocol used to perform the crystallization studies, in this specific case a protocol where the shear pulse is given at $170{ }^{\circ} \mathrm{C}$. The figure on the right displays the cooling profile measured during the cooling step performed after the shear pulse. The black lines indicate the average value of 20 cooling runs, whereas the red lines display the standard deviation. 

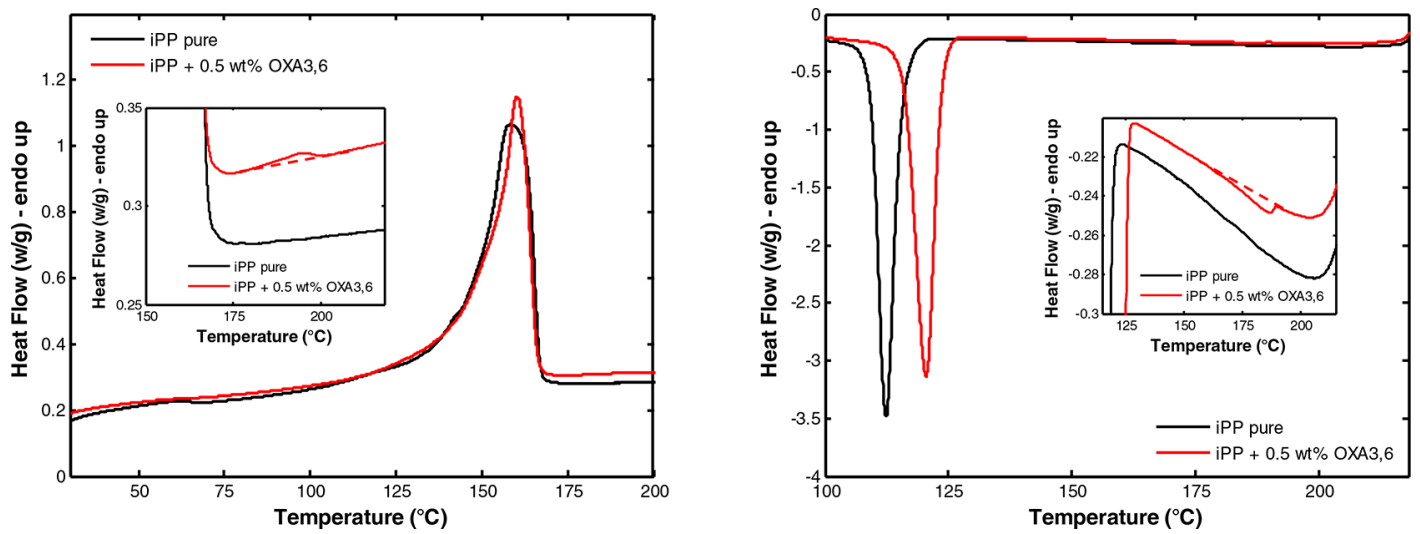

Figure 2. DSC heating (left) and cooling (right) traces showing the melting and crystallization of both the $i \mathrm{PP}$ and OXA3,6 (insets). The heating and cooling rates during these experiments were $10{ }^{\circ} \mathrm{C} / \mathrm{min}$.

after equilibration of the sample at the set temperature. Figure 1 on the left shows a schematic overview of the applied experimental protocol, whereas Figure 1 on the right depicts the temperature of the sample measured during the crystallization step. It should be noted that the temperature profile measured is at the location where the Xray beam passes the MPR sample.

Molecular Modeling. Molecular modeling was employed to investigate whether the $i \mathrm{PP}$ sample with OXA3,6 exhibits an unusual flow response. In this respect, we have used the tube model of Das et $\mathrm{al}^{22}$ The model incorporates the molecular mechanisms of reptation, contour length fluctuations, constraint release, and Rouse motions. The interested reader is referred to the Supporting Information and the original article for further information. ${ }^{22}$ To predict the linear viscoelastic response of a polymer melt, i.e., the dynamic moduli $G^{\prime}$ and $G^{\prime \prime}$ as well as the complex viscosity $\eta^{*}$, the algorithm requires the use of two parameters only, namely, the entanglement molecular weight, $M_{\mathrm{e}}$, and the entanglement relaxation time, $\tau_{\mathrm{e}}$. According to tube theory, ${ }^{22-25}$ the values of those parameters are independent of the molecular weight distribution (MWD) and average molecular weight, meaning that they should be the same for both the pure $i \mathrm{PP}$ and the $i$ PP melt blended with OXA3,6. In this respect, we have used the values $M_{\mathrm{e}}=5 \mathrm{~kg} / \mathrm{mol}$, and $\tau_{\mathrm{e}}=2 \times 10^{-7} \mathrm{~s}$ for both samples. For verification of the model used and the parameters used to describe the experimental data of the samples reported in this study, data fitting of other $i \mathrm{PP}$ samples from the literature was performed. The experimental data for three different $i \mathrm{PP}$ samples reported by Hamad et al. ${ }^{26}$ and Eckstein et al. ${ }^{27}$ with various polydispersity and molecular weights were successfully described with the model, as can readily be observed from Figure 8. Additional information on this verification procedure is provided in the Supporting Information.

Considering the density $\rho=0.75 \mathrm{~g} / \mathrm{cm}^{3}$ at $170{ }^{\circ} \mathrm{C},{ }^{27}$ the value for $M_{\mathrm{e}}$ gives a plateau modulus $G_{0}=0.44 \mathrm{MPa}$ since $G_{0}=4 \rho R T /\left(5 M_{\mathrm{e}}\right)$, where $R$ is the gas constant and $T$ the temperature. Apart from the two aforementioned parameters, the model requires the MWDs of the samples. Here, we have used the experimental distributions, as determined from the GPC measurements. The average molecular weights inferred from the GPC data are $M_{\mathrm{w}}=430$ and $400 \mathrm{~kg} / \mathrm{mol}$ for the pure $i \mathrm{PP}$ and the $i \mathrm{PP}$ blended with OXA3,6, respectively. The corresponding polydispersity indexes are respectively $\mathrm{PDI}=4.2$ and 4.5.

Characterization Methods. Crystallization and melting behavior was determined using DSC analysis performed using a TA Instruments Q2000 DSC. All samples were measured at heating and cooling rates of $10^{\circ} \mathrm{C} / \mathrm{min}$ up to a maximum temperature of 220 ${ }^{\circ} \mathrm{C}$.

The MPR bars obtained after experiments were cut into $15 \mu \mathrm{m}$ thick slices using a Lyca RM2235 microtome. Samples were continuously cooled using liquid nitrogen during the microtomy process. Polarization optical micrographs depicting the morphology of the slices were taken between cross-polarizers on an Olympus BX53 microscope mounted with an Olympus DP26 camera.
Polarization optical micrographs (between cross-polarizers and using a $530 \mathrm{~nm} \lambda$-wave plate) were taken on an Olympus BX53 microscope mounted with an Olympus DP26 camera and a Linkam hotstage. The samples were heated to $200{ }^{\circ} \mathrm{C}$ at a rate of $30^{\circ} \mathrm{C} / \mathrm{min}$. After leaving the samples for $5 \mathrm{~min}$ in this isothermal condition, the samples were cooled at a specified cooling rate to $170{ }^{\circ} \mathrm{C}$ to induce the crystallization of OXA3,6 crystallites. Subsequently, the samples were cooled at a rate of $30{ }^{\circ} \mathrm{C} / \mathrm{min}$ to $135{ }^{\circ} \mathrm{C}$ where the morphological changes during isothermal crystallization were monitored.

Online 2D small-angle X-ray scattering (SAXS) experiments (wavelength $=0.1 \mathrm{~nm}$ ), following the crystallization process during and after the flow experiments, were performed at the DUBBLE beamline (BM26B) at the European Synchrotron Radiation Facility (ESRF) in Grenoble, France. As is reported by Bras et al. ${ }^{28}$ and Portale et al., ${ }^{29}$ this beamline is particularly optimized for polymer science. During the shear pulse and crystallization process, SAXS images were collected with a 2D Pilatus $1 \mathrm{M}$ detector $(169 \mathrm{~mm} \times 179$ $\mathrm{mm}$ active area) placed at $6.47 \mathrm{~m}$ distance from the sample. The detector was triggered by an electric TTL pulse at the start of the displacement of the pistons in order to synchronize the data acquisition. The data were background corrected and normalized for synchrotron beam fluctuations using an ionization chamber placed before the sample. Furthermore, correction for the sample absorption was performed using a photodiode located at the beamstop. A standard rat tail tendon collagen fiber was used to calibrate the modulus of the scattering vector $q$-scale, using the position from diffraction peaks where $q=4 \pi \sin \theta / \lambda$ with $\theta$ being half of the scattering angle. The maximum value in the Lorentz corrected 1DSAXS pattern, obtained after integrating the meridional and diagonal scattering intensities, was identified as the long period $\left(L_{\mathrm{p}}\right)$ of the samples and was calculated using $d=2 \pi / q$. The Lorentz correction has been applied through multiplying the measured intensity distribution by a factor $q$ for the meridional scattering intensity (oriented crystallites).

The linear viscoelastic behavior $\left(G^{\prime}\right.$ and $\left.G^{\prime \prime}\right)$ of the samples was measured using an Anton Paar MCR 702 Twindrive $\left(170^{\circ} \mathrm{C}\right)$ with parallel plate geometry.

The molecular weight and polydispersity were studied by size exclusion chromatography (SEC) measurements performed at $150{ }^{\circ} \mathrm{C}$ on a Polymer Char GPC-IR built around an Agilent GC oven model 7890, equipped with an autosampler and the integrated detector IR4. 1,2-Dichlorobenzene (oDCB) was used as an eluent at a flow rate of 1 $\mathrm{mL} \mathrm{min}^{-1}$. The SEC data were processed using Calculations Software GPC One. The molecular weights distributions were calculated with respect to polyethylene standards.

\section{RESULTS AND DISCUSSION}

Effect of OXA3,6 on iPP Melt and Crystallization Behavior. The characteristic effect of oxalamide based 

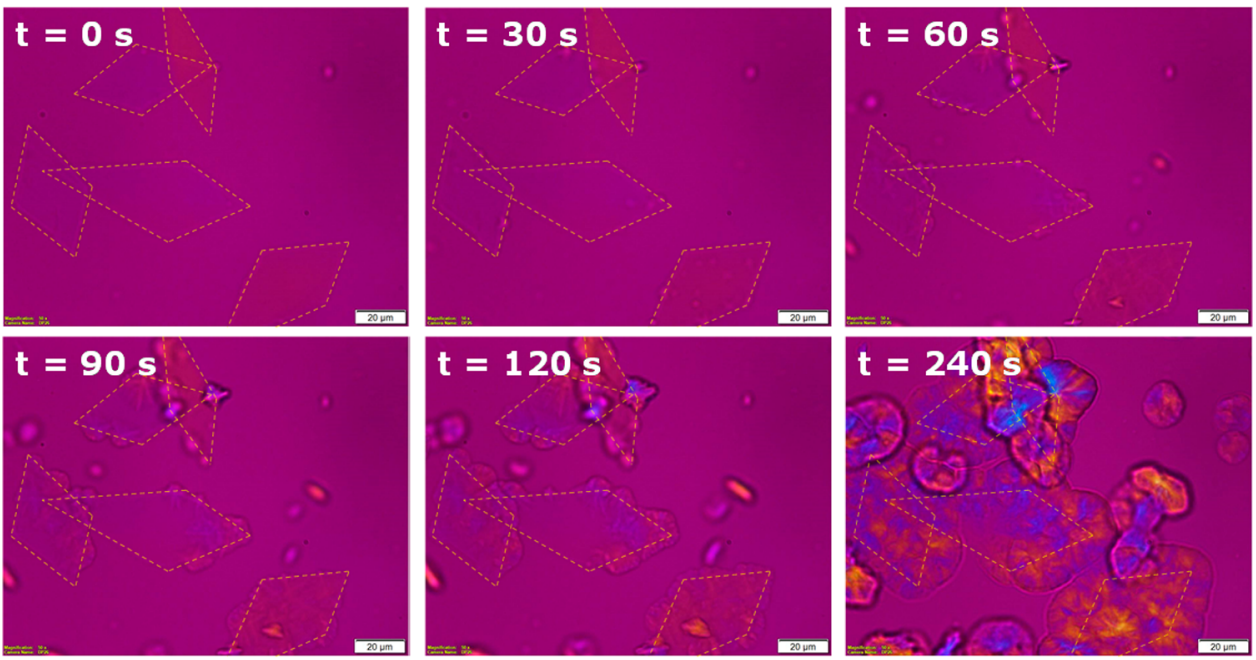

Figure 3. Polarization optical micrographs taken during isothermal crystallization of $i \mathrm{PP}$ in the presence of 0.5 wt \% OXA3,6. The sample was heated to $200{ }^{\circ} \mathrm{C}$ and left isothermal for $5 \mathrm{~min}$ to ensure all NA particles were molten. Next, the sample was cooled at a rate of $1{ }^{\circ} \mathrm{C} / \mathrm{min}$ to $170{ }^{\circ} \mathrm{C}$ to allow the NA to crystallize into large rhombic aggregates. After reaching $170{ }^{\circ} \mathrm{C}$ the sample was cooled at a rate of $30^{\circ} \mathrm{C} / \mathrm{min}$ to $135{ }^{\circ} \mathrm{C}$, after which the crystallization morphology was monitored over time. Please note that due to the poor visibility of the NA particles, yellow dotted lines are placed over borders of the NA aggregates to guide the eye.
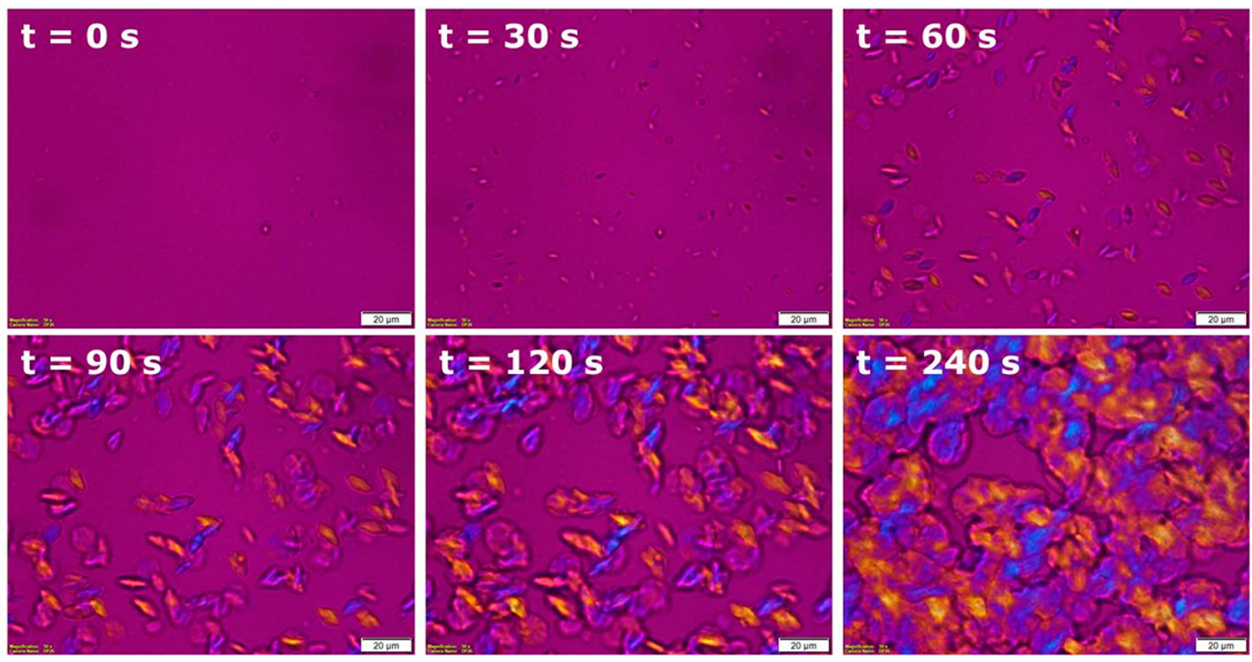

Figure 4. Polarization optical micrographs taken during isothermal crystallization of $i \mathrm{PP}$ in the presence of 0.5 wt \% OXA3,6. The sample temperature profile was adopted as described in Figure 3, except that the sample was cooled at a rate of $30{ }^{\circ} \mathrm{C} / \mathrm{min}$ from 200 to $170{ }^{\circ} \mathrm{C}$ to allow the NA to crystallize into fine aggregates. The displayed polarization optical micrographs were taken during isothermal crystallization, after consecutive cooling to $135^{\circ} \mathrm{C}$.

nucleating agent (NA) OXA3,6 on the melting and crystallization behavior of $i \mathrm{PP}$ as observed from DSC is depicted in Figure 2. Upon heating, first the melting endotherm of the $i \mathrm{PP}$ crystallites is observed at $165{ }^{\circ} \mathrm{C}$. Upon further heating, a broad but low enthalpy endotherm is observed around $190{ }^{\circ} \mathrm{C}$ in the presence of OXA3,6, which corresponds to the melting of the NA crystals (Figure 2, left inset). Upon consecutive cooling, first the crystallization exotherm of the nucleating agent is observed starting at 190 ${ }^{\circ} \mathrm{C}$, followed by the exotherm of the $i \mathrm{PP}$ crystallization with a peak crystallization temperature $\left(T_{c}\right)$ at $123{ }^{\circ} \mathrm{C}$ (Figure 2, right). These DSC traces confirm the nucleating ability of OXA3,6, as its presence results in a rough $10^{\circ} \mathrm{C}$ increase in $T_{\mathcal{O}}$ since pure $i \mathrm{PP}$ processed under the same conditions displays a $T_{\mathrm{c}}$ at $112^{\circ} \mathrm{C}$.

From Figure 2 it is apparent that both iPP and OXA3,6 crystallites are molten at or above $200{ }^{\circ} \mathrm{C}$. As is reported for
OXA3,6/iPP systems, the melting and crystallization temperature of OXA3,6 in iPP decreases with decreasing concentrations. ${ }^{4}$ Such a melting temperature suppression is characteristic for compatible systems, indicating that $i \mathrm{PP}$ acts as a solvent for OXA3,6 at elevated temperatures. It should be noted that it is difficult to estimate a useful interaction parameter for the $i \mathrm{PP} / \mathrm{OXA3,6}$ system due to the different polarity and interactions of the hexane end-groups and the central hydrogen-bonding oxalamide motifs of the OXA3,6 molecule. That being said, considering that the Hildebrand interaction parameters of $n$-hexane $\left(\delta=14.7\left(\mathrm{MJ} / \mathrm{m}^{3}\right)^{1 / 2}\right)$ and iPP $\left(16.7\left(\mathrm{MJ} / \mathrm{m}^{3}\right)^{1 / 2}\right)$ are relatively close, combined with the knowledge that polypropylene can absorb $\sim 9$ wt $\% n$-hexane already at room temperature, ${ }^{30}$ we expect that the hexane endgroups of OXA3,6 will interact/mix with $i \mathrm{PP}$ above its melting temperature. 

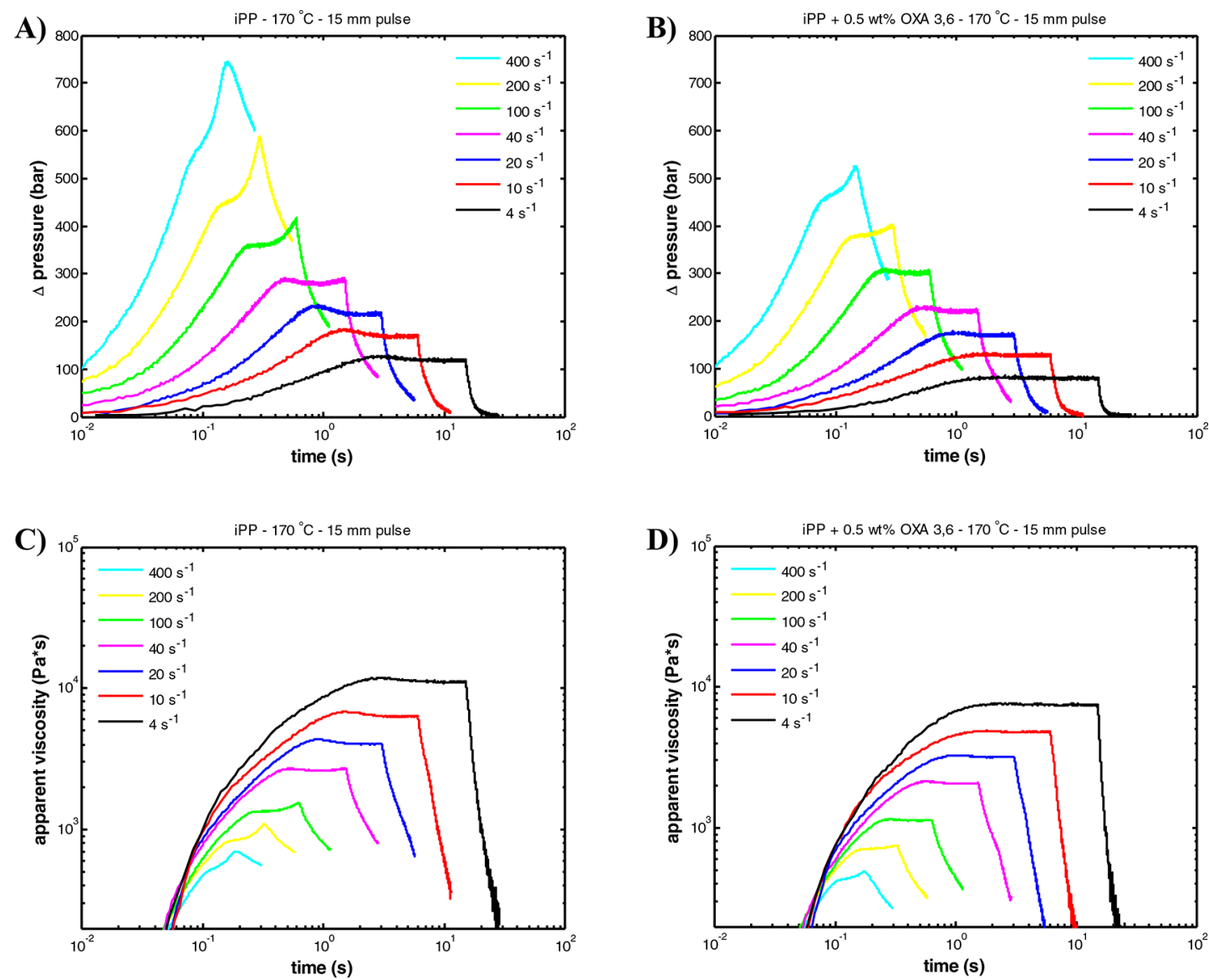

Figure 5. Pressure difference versus time observed during slit-flow experiments performed on pure $i \mathrm{PP}$ (A) and iPP containing 0.5 wt $\%$ OXA3,6 (B). Similarly, (C) and D) depict the apparent viscosity versus time obtained after converting the data using eqs $1-3$.

Though the nucleating ability of OXA3,6 is clear from the data displayed in Figure 2, we would like to highlight that the NA particle morphology generated during cooling has a significant influence on the observed nucleating efficiency. For example, cooling an $i \mathrm{PP}$ melt with 0.5 wt \% OXA3,6 at a rate of $1{ }^{\circ} \mathrm{C} / \mathrm{min}$ from 200 to $170{ }^{\circ} \mathrm{C}$, the temperature range where crystallization of OXA3,6 occurs at this concentration, provides ample time to grow thin rhombic particles of more than $50 \mu \mathrm{m}$ in diagonal length. As is evident from Figure 3, subsequent cooling to $135{ }^{\circ} \mathrm{C}$ results in $i \mathrm{PP}$ nucleation and crystal growth on the surface of the generated NA particles. Please note that the depicted NA particles in Figure 3 are expected to be very thin given their weak birefringent behavior in polarized optical microscopy (POM). To improve their visibility, yellow dotted lines are placed over the borders of the NA particles in all micrographs of Figure 3.

In contrast, rapid cooling of $i \mathrm{PP}$ with 0.5 wt \% OXA3,6 from 200 to $170{ }^{\circ} \mathrm{C}$ results in the formation of a high number of very small NA aggregates that are barely detectable by POM, indicating they are (sub)micrometer sized. Upon further cooling, followed by isothermal crystallization at $135{ }^{\circ} \mathrm{C}$, a high number of $i \mathrm{PP}$ crystallites and crystals are formed as a result of the fine distribution of NA particles, as is shown in Figure 4. When comparing the trends depicted in Figures 3 and 4, one can conclude that an increasing cooling rate decreases the NA particle size, yielding a more homogeneous NA distribution and a more homogeneous $i \mathrm{PP}$ nucleation and crystal growth. In other words, the overall nucleating efficiency is dependent on the thermal history and favors high cooling rates.

In addition to this effect of cooling rate, OXA3,6 and other oxalamide based NAs are known to exhibit a further enhancement in nucleation efficiency after the application of shear. ${ }^{31}$ For example, in previous work we demonstrated that shearing iPP systems blended with OXA3,6 increased the onset of crystallization from 123 to $140{ }^{\circ} \mathrm{C}$, making it comparable to other organic nucleating agents such as the sorbitol derived nucleating agent DMDBS. ${ }^{4}$ This synergetic effect between the nucleating efficiency of OXA 3,6 and shear is believed to be similar to that of sheared systems that contain (nucleating) nanoparticles. ${ }^{15,19,32-36}$ In general, this synergy is attributed to the enhancement of the flow effect on the polymer kinetics and morphology. ${ }^{37}$ As a result, the stress is locally enhanced and the chain mobility decreased via anchoring of the polymeric chains on the particle surface. This phenomenon is often reported for systems that contain well-defined solid inorganic fillers. Even though the crystal morphology of OXA3,6 particles is influenced by both cooling and shear protocols, the resulting (sub)micrometer dimensions generated at high cooling rates could enhance crystallization after shear in a similar fashion as inorganic solid fillers.

To identify the exact role of shear on both the $i \mathrm{PP}$ melt and the OXA3,6 morphology, shear experiments were performed at a temperature of $170{ }^{\circ} \mathrm{C}$, a temperature where self-assembly of the NA proceeds as is evident from the right inset in Figure 2. After keeping the samples isothermal for $5 \mathrm{~min}$ to ensure all OXA3,6 has crystallized, a shear pulse of $15 \mathrm{~mm}$ was applied. 
The full procedure for the preparation of the sample bars and the procedures used for the MPR experiments are reported in the Experimental Section. The data are converted to apparent wall shear rate $(\dot{\gamma})$ and apparent viscosity $(\eta)$ using eqs $1-3$ :

$$
\begin{aligned}
& \dot{\gamma}=\frac{6 Q}{W H^{2}} \\
& \sigma=\frac{H \Delta P}{2\left(1+\frac{H}{W}\right) L} \\
& \eta=\frac{\sigma}{\dot{\gamma}}=\frac{H^{2}}{12\left(1+\frac{H}{W}\right) L} \frac{\Delta P}{V_{\text {piston }}}
\end{aligned}
$$

where $Q$ is the volumetric flow rate being $H \times W \times V_{\text {piston }}$ and $W$ and $H$ are the slit width and thickness of 6 and $1.5 \mathrm{~mm}$, respectively. ${ }^{38}$ For this specific setup $L$ is $120 \mathrm{~mm}$, being the distance between the transducers. The pressure difference $(\Delta P)$ between the two pressure transducers was monitored over time after varying the piston speeds $\left(V_{\text {piston }}\right)$ from 1 to 100 $\mathrm{mm} / \mathrm{s}$, translating into $\dot{\gamma}$ between 4 and $400 \mathrm{~s}^{-1}$ following eq 1 .

Figure 5 displays the evolution of both the pressure difference (A and B) and the apparent viscosity (C and $D$ ) as a function of $\dot{\gamma}$ for the pure iPP and the iPP sample blended with OXA3,6. For low $\dot{\gamma}$ the viscosity builds up during the pulse until it reaches a steady wall shear stress, indicated by the plateau value. After the pulse ends, the pressure difference drops rapidly due to relaxation of the melt. As is clearly visible from the pure $i \mathrm{PP}$ sample (Figure 5C), when applying a pulse with $\dot{\gamma} \geq 20 \mathrm{~s}^{-1}$, a gradual increase in apparent viscosity is observed after reaching the steady shear plateau. This behavior is generally attributed to the formation of shear-induced precursors and their consecutive crystallization under the elevated pressures. ${ }^{21}$ Though the behavior for the system containing OXA3,6 is comparable to the shear behavior of the pure $i \mathrm{PP}$ sample, two characteristic differences can be observed. First, the plateau levels of the viscosity are lower than those for the pure $i \mathrm{PP}$ at the same shear rates (Figure 6). Second, the formation and crystallization of shear-induced precursors at the wall seems to require significantly higher shear rates. As is visible from Figure 5B, a deviation from the

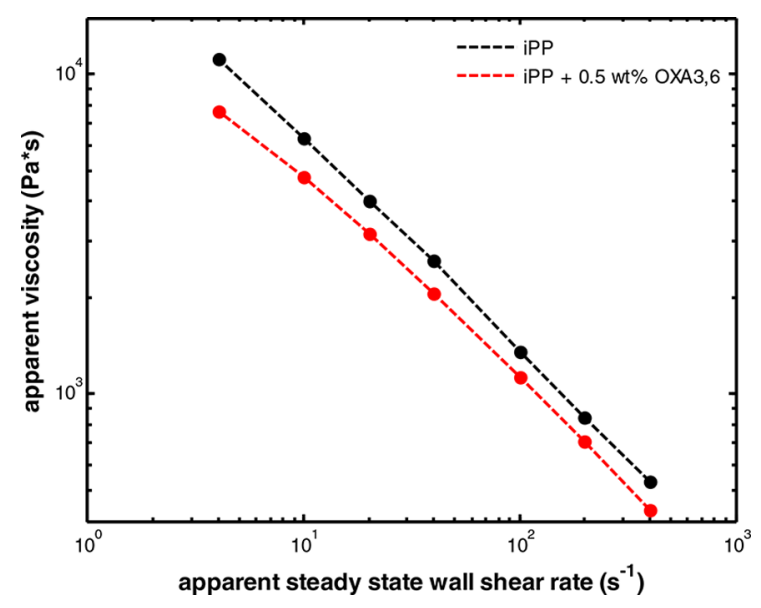

Figure 6. Apparent steady shear viscosity $(\eta)$ versus steady state wall shear rate $(\dot{\gamma})$ for pure $i \mathrm{PP}$ and $i \mathrm{PP}$ containing $0.5 \mathrm{wt} \%$ OXA3,6, extracted from the MPR slit-flow experiments depicted in Figures $5 \mathrm{C}, \mathrm{D}$. plateau pressure is only observed at $\dot{\gamma}=100 \mathrm{~s}^{-1}$ and is only clearly evident at $\dot{\gamma} \geq 200 \mathrm{~s}^{-1}$ and higher. These findings indicate that the sample containing OXA3,6 has a lowered viscosity, which could be linked to the molecular weight $\left(M_{\mathrm{w}}\right)$.

To identify whether the sample containing OXA3,6 has a lowered viscosity due to a lower molecular weight, we aimed to verify that no degradation has occurred during the processing steps. For this reason, both samples have been analyzed using high-temperature size-exclusion chromatography (Figure 7A). The SEC analysis shows that the molecular weight distribution and $M_{\mathrm{w}}$ values of the samples are comparable. The $i \mathrm{PP}$ blended with OXA3,6 was found to have a $M_{\mathrm{w}}$ of $400 \mathrm{~kg} / \mathrm{mol}$ (PDI = 4.5 ), while the pure $i$ PP sample has a $M_{\mathrm{w}}$ of $430 \mathrm{~kg} / \mathrm{mol}$ (PDI $=4.2$ ). Indeed, this indicates that the processing into MPR bars results in a slight variation in $M_{w}$, but when considering linear chains this difference is not significant enough to explain the observed suppression in apparent shear viscosity depicted in Figure 6.

Moreover, comparison of the MPR data with data obtained from rheology performed at $170{ }^{\circ} \mathrm{C}$ confirms the reduction of the viscosity of $i \mathrm{PP}$ upon the introduction of OXA3,6 (Figure $7 \mathrm{~B})$. When comparing the complex viscosity versus frequency from rheometry with the apparent viscosity versus apparent shear rate from the MPR shear experiments, we observe that the data from both experiments are in rather good agreement. This indicates that the empirical Cox-Merz rule, $\eta(\dot{\gamma})$ $=\eta^{*}(\omega)$, holds for both samples. ${ }^{39}$ More importantly, this indicates that the viscosity difference between the two samples is consistently observed with both experimental methods. Furthermore, although the pure $i \mathrm{PP}$ sample has not reached a plateau value for the complex viscosity, we can readily observe that its plateau value is roughly 3 times higher than that of $i \mathrm{PP}$ blended with OXA3,6. Such a suppression in plateau viscosity corresponds to a difference in molecular weight $\left(M_{\mathrm{w}}\right)$ of approximately $30 \%$ (using the relation $\eta \sim M_{\mathrm{w}}{ }^{3.4}$ for entangled linear polymer chains), while the findings from SEC analysis can only account for a molecular weight reduction of $7 \%$. The suppression in viscosity of $i \mathrm{PP}$ in the presence of OXA3,6 is repeatedly observed, also when varying the OXA3,6 concentration, as is described in the Supporting Information (Figure S8).

To obtain more information on this discrepancy in melt behavior, the GPC traces have been used to predict and verify the melt response using the tube model. From the fits obtained (Figures 7C,D) it is evident that the model provides only a good fit to the pure iPP melt while the model fails clearly for iPP blended with OXA3,6. In particular, it grossly overestimates the magnitude of the dynamic moduli, meaning that the actual molecular relaxation is much faster than the model predicts.

Even though the NA is present as a solid at the used experimental temperatures, it is not possible to explain this strong disagreement between the tube model and the experimental data by considering that the NA would be present in the molten state and acts as solvent. This is because the weight fraction of the NA is far too low to produce such a dramatic speed up of the molecular relaxation. The same applies for the low- $M_{\mathrm{w}}$ tail of the molecular weight distribution; considering all fractions, the maximum volume fraction of unentangled chains is 0.03 .

One possible explanation for the observed suppression in viscosity could be related to the topological architecture of the used $i \mathrm{PP}$ grade. For example, the used $i \mathrm{PP}$ batch could contain 

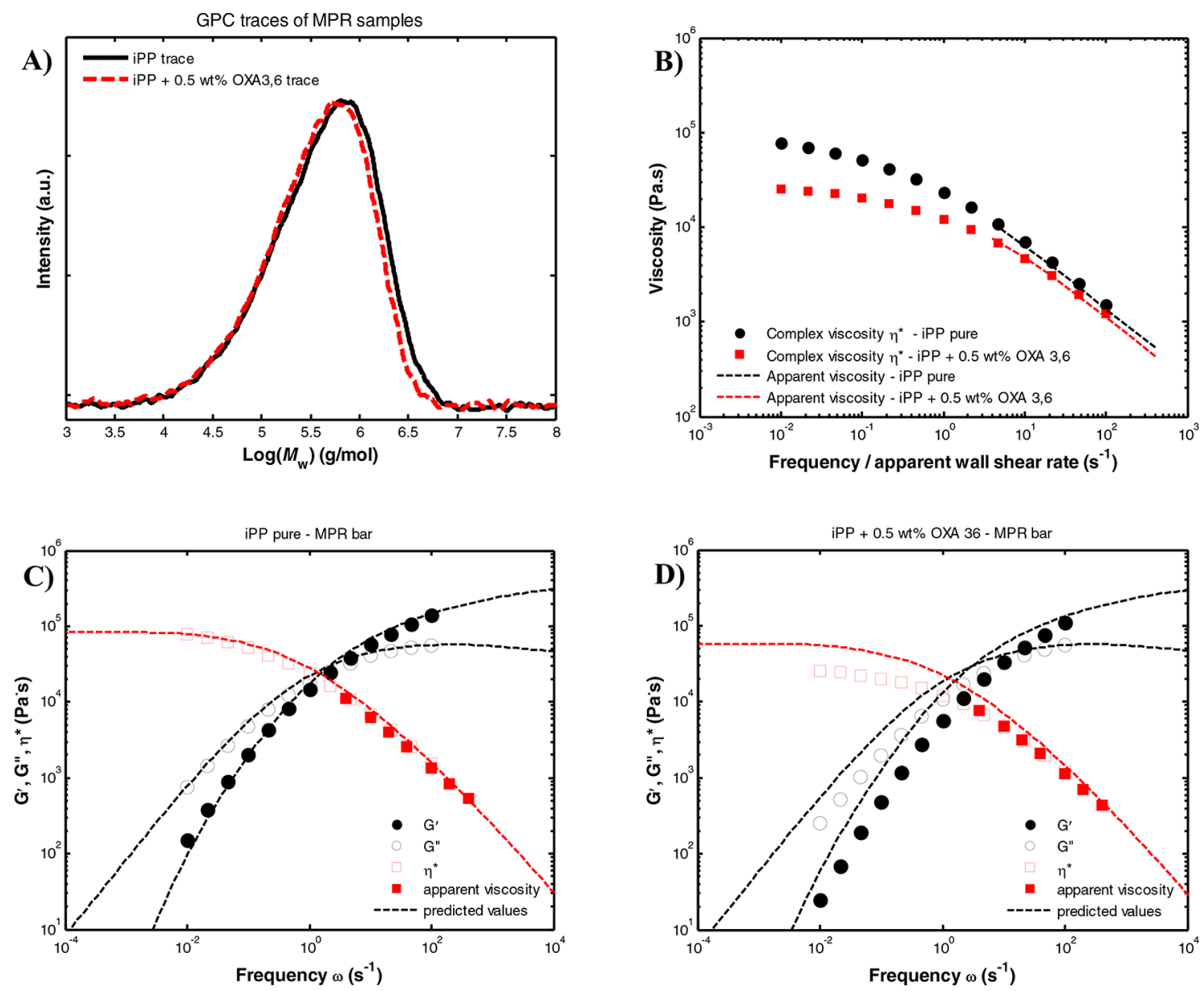

Figure 7. (A) GPC traces of the pure $i \mathrm{PP}$ and nucleated $i \mathrm{PP}$ samples. (B) Apparent viscosity observed as a function of apparent wall shear rate during steady shear together with the complex viscosity over frequency observed from rheology at $170{ }^{\circ} \mathrm{C}$. Images (C) and (D) depict the experimental and predicted values of the elastic modulus $\left(G^{\prime}\right.$ in $\left.\mathrm{Pa}\right)$, viscous modulus $\left(G^{\prime \prime}\right.$ in $\left.\mathrm{Pa}\right)$, and complex viscosity $\left(\eta^{*}\right)$ obtained from the tube model for pure $i \mathrm{PP}$ and nucleated $i \mathrm{PP}$, respectively.

a small fraction of long chain branches, dependent on the used synthesis protocol and catalyst. If this would be the case, chain scission (degradation) of these branches could occur during processing which would have a significant effect on the melt viscosity. This is because, for branched molecules, the dependence between zero-shear viscosity and molecular weight is exponential rather than linear as for linear chains. ${ }^{22-25}$ Although unlikely, the possibility that the used iPP grade contains a minor fraction of long chain branches cannot be excluded. However, we believe it is extremely unlikely that the observed viscosity difference between the pure $i \mathrm{PP}$ and the $i \mathrm{PP}$ blended with OXA3,6 is resulting from a variation in topology, i.e., degradation of long chain branches. Our argument supporting this statement is threefold:

First, using the same tube model and parametrization as the one used to obtain the predictions mentioned above, we have examined the possibility that the viscosity suppression of the $i$ PP with OXA3,6 originates from degradation of long chain branches. In more detail, we have considered a small weight fraction of long chain branching (either 0.1 or 0.2 ) to be present in the parent $i \mathrm{PP}$ batch. Further, assuming that such branches undergo degradation in the iPP melt with OXA3,6, we have found that the rheological response of the blend (linear chains and degraded branches) never falls below the rheological response of the system without branching, i.e., the polydisperse melt of linear chains, the response of which is shown in Figure 7D. This behavior is consistent even if we consider severe degradation of the branched molecules (Figures S4, S5, and S7). The interested reader is referred to the Supporting Information for more details concerning our calculations and findings.

Second, if the iPP grade used in this study contained longchain branches, it would have been impossible to describe the viscoelastic response of known linear $i \mathrm{PP}$ grades found in the literature using the same parametrization as the one used herein. Such an exercise was performed on two commercial $i \mathrm{PP}$ grades $^{26}$ (CiPP3 and CiPP5) and on iPP from a metallocene catalyst $^{27}$ (sample M9), which were all reported to be linear in topology. We observe that the viscoelastic response of all samples could be described rather well with the used parameters including the $i \mathrm{PP}$ grade used in this study (Figure 8 ). Especially the agreement between the predicted and observed viscoelastic response along the terminal regime is strong evidence that all evaluated $i \mathrm{PP}$ grades do not contain long-chain branches as this would result in deviations in the elastic response at low frequencies.

Third, even if the used $\mathrm{PP}$ grade would be branched, both the pure $i \mathrm{PP}$ and the $i \mathrm{PP}$ blended with OXA3,6 have been prepared under the same processing conditions, which should result in a comparable degree of chain scission (degradation). Therefore, we would anticipate strong deviations between theoretical predictions and experimental data to occur in both 

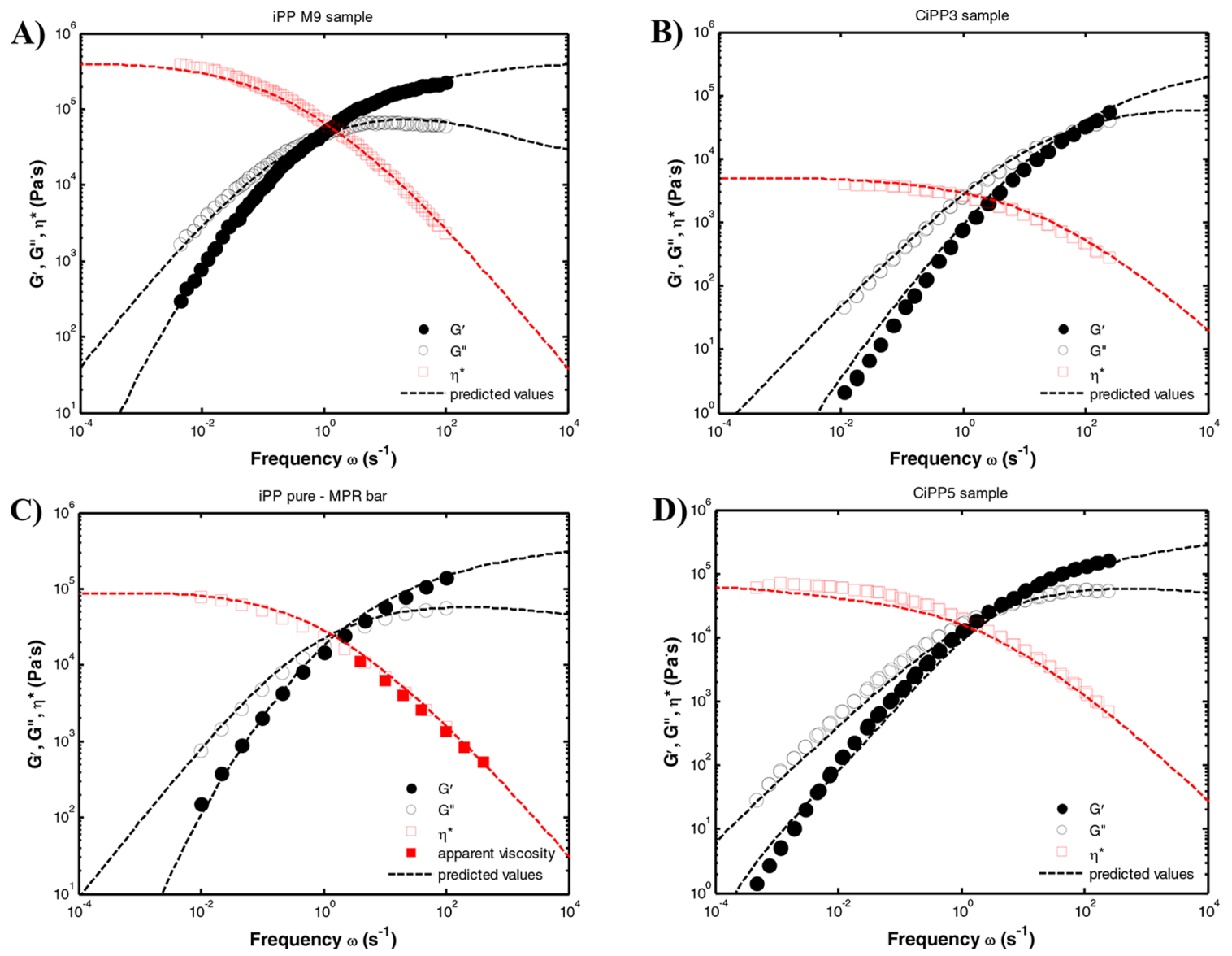

Figure 8. Predicted values of the storage modulus $\left(G^{\prime}\right.$ in $\left.\mathrm{Pa}\right)$, loss modulus $\left(G^{\prime \prime}\right.$ in $\left.\mathrm{Pa}\right)$, and complex viscosity $\left(\eta^{*}\right)$ obtained from the BOB model using the GPC traces as input for (A) iPPM9, (B) CiPP3, (C) iPP pure (this work), and (D) CiPP5. Model predictions correspond to $\rho=0.75 \mathrm{~g} /$ $\mathrm{cm}^{3}, T=170{ }^{\circ} \mathrm{C}, M_{\mathrm{e}}=5 \mathrm{~kg} / \mathrm{mol}, \tau_{\mathrm{e}}=2 \times 10^{-7} \mathrm{~s}$, monomeric mass $M_{0}=42.08(\mathrm{~g} / \mathrm{mol})$, and dilation exponent $\alpha=1$.

grades, not only in the $i \mathrm{PP}$ grade blended with OXA3,6 (which is the current case).

A more realistic explanation for the aforementioned viscosity suppression in iPP blended with OXA3,6 is that the OXA3,6 interacts with the melt through physical absorption. ${ }^{40,41}$ Since the OXA3,6 dissolves in $i \mathrm{PP}$ at elevated temperatures, it is expected that the hexane end-groups of OXA3,6 interact with the $i$ PP chain segments in the random coil. Upon cooling and consecutive crystallization of OXA3,6, driven by the formation of optimal hydrogen bonding between the oxalamide moieties, the mobility of the hexane end-groups is hindered (compared to their melt state). ${ }^{8}$ Retrospectively, the interaction of $i \mathrm{PP}$ chains with the end-groups of OXA3,6 is anticipated to facilitate physical absorption and local immobilization of the interacting $i \mathrm{PP}$ segments. Within the experimental time frame, given that the high molecular weight chains have a larger radius of gyration, the longer chains are more likely to have chain segments interacting with the nucleating agent. The absorbed chain segments prevent reptation of the remainder of the chain which can therefore be considered immobilized, at least within the given experimental time scales. ${ }^{42}$ The anticipated absorption of the $i \mathrm{PP}$ chains is schematically represented in Figure 9. In general, three routes for the physical absorption can be envisioned: (1) physical absorption of a few segments of $i$ PP chains, giving rise to long dangling chain-ends; (2) physical absorption of most of the $i \mathrm{PP}$ chain, thereby withdrawing it from the $i \mathrm{PP}$ melt; and (3) a combination of the first two resulting in short dangling chain-ends.

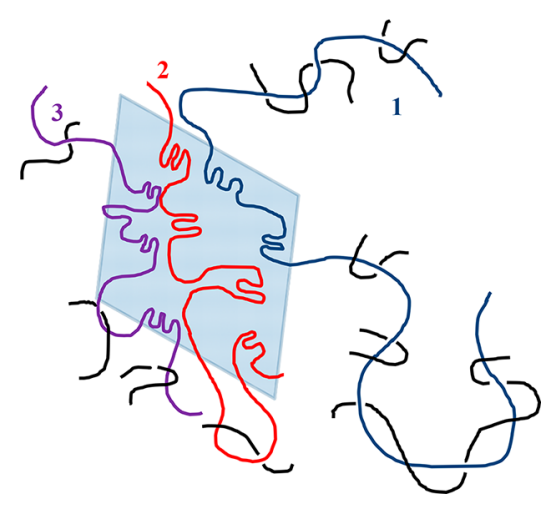

Figure 9. Schematic representation of possible topological states of physical absorption: (1) absorption of a few iPP segments resulting in long dangling chain-ends interacting with the melt; (2) absorption of many segments in the $i \mathrm{PP}$ chain, effectively withdrawing it from the melt; and (3) a combination of (1) and (2) yielding short dangling chain-ends.

The first route enforces the situation encountered at systems where reptative chain dynamics is severely suppressed, leading to an increased elastic contribution. In such systems, e.g. melts of semicrystalline chains or melts of associating chains, the molecular origin of the aforementioned behavior is attributed to contour length fluctuations of long dangling chain-ends ${ }^{43}$ or delayed (sticky) reptation dynamics. ${ }^{44-46}$ In contrast, the second route can lead to an effective suppression of the melt 

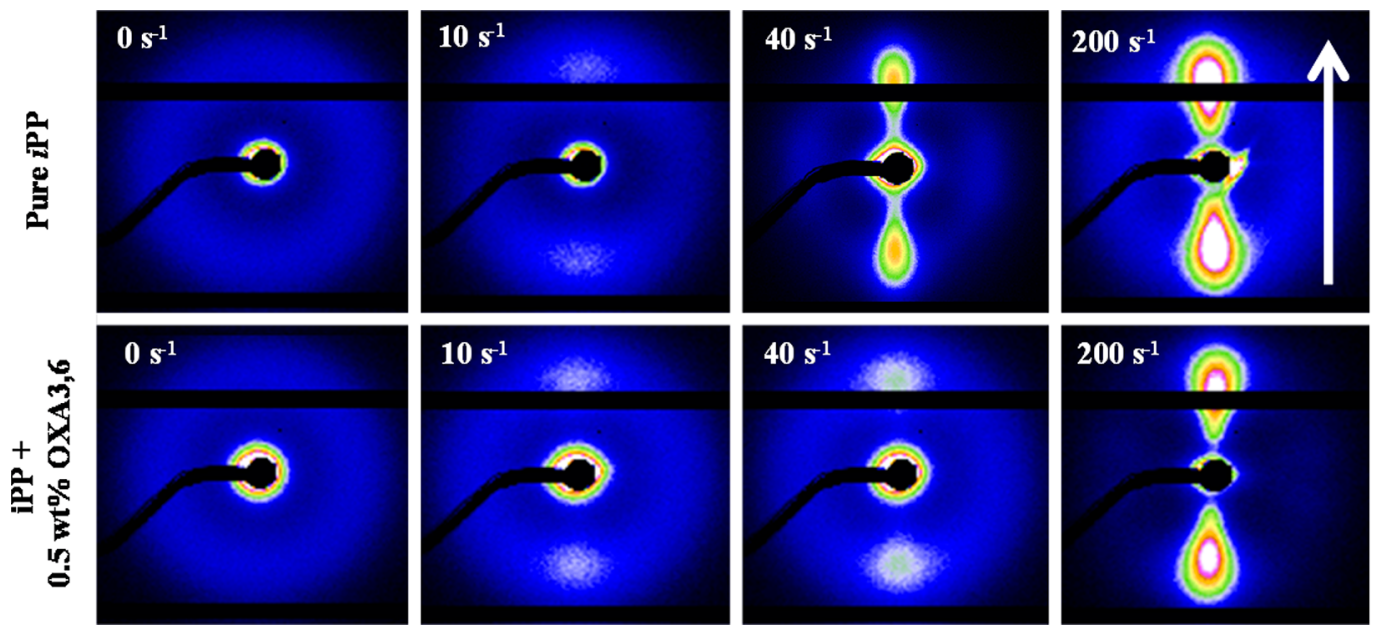

Figure 10. 2D SAXS patterns obtained after cooling and crystallization at $100{ }^{\circ} \mathrm{C}$ of both pure $i \mathrm{PP}$ and $i \mathrm{PP}$ containing 0.5 wt $\%$ OXA3,6 after various shear conditions. The arrow depicts the flow direction of the shear pulses.

viscosity since the removal of long chains from the bulk increases the entanglement molecular weight and, in turn, speeds up the entanglement relaxation time, effectively resulting in a lowered viscosity of the $i \mathrm{PP}$ bulk. Indeed, this kind of behavior has been observed in nanoparticle-filled iPP polymer melts, provided that the interparticle distance is not less than the radius of gyration of the chains as for the bridging effect to take place. ${ }^{20}$ It is worth emphasizing that the $i$ PP melt blended with OXA3,6 fulfills this condition. Further, we expect both mechanisms (routes) to occur, as is the case in scenario 3, with the net effect of those two opposing mechanisms being a suppression of the melt viscosity. According to this hypothesis, the absorption of high molecular weight chains on the OXA3,6 crystal surface and their (partial) withdrawal from the $i \mathrm{PP}$ melt will result in the remaining iPP bulk to (1) have a lower average molecular weight (2) relax faster, and (3) exhibit a lowered viscosity. ${ }^{20,42,47-49}$ Indeed, the tube model could capture the data of the $i \mathrm{PP}$ with OXA3,6 by considering a higher $M_{\mathrm{e}}$ value and a faster $\tau_{\mathrm{e}}$. Nevertheless, the model clearly fails when it considers a homogeneous melt parametrized by the same $M_{\mathrm{e}}$ and $\tau_{\mathrm{e}}$ as the pure $i \mathrm{PP}$. (At present, all tube models assume a homogeneous melt. $\left.{ }^{22-25,50-52}\right)$

From the findings in the previous section, one would expect that the decrease in melt viscosity of $i \mathrm{PP}$ in the presence of OXA3,6 is lost upon melting of the NA crystallites, as they are presumed to dissolve molecularly upon melting. However, preliminary findings indicate that this is not the case, as is observed from slit-flow experiments at $200{ }^{\circ} \mathrm{C}$, as is described in the Supporting Information (Figure S9). The fact that OXA3,6 still lowers the melt viscosity of the system after its crystals are molten gives rise to the hypothesis that the NA does not fully dissolve in the $\mathrm{PP}$ melt but instead persists as small clusters held together by weak hydrogen bonding between the oxalamide motiffs, while the hexane end-groups of OXA3,6 are interacting with/dissolved in the $i \mathrm{PP}$ melt. If such clusters persist in the molten state, they could still facilitate the absorption of $i \mathrm{PP}$ chains and maintain the suppression of the $i \mathrm{PP}$ melt viscosity. Such behavior would explain why the suppression in zero-shear viscosity fluctuates with both concentration and thermal history of the sample, as both factors strongly influence the NA dispersion in the iPP melt and govern the particle size. Verification of this hypothesis is part of ongoing research, but as it requires analysis of blends with various $i \mathrm{PP}$ grades and various NAs and their concentrations to control the viscosity ratio and to influence the size and dispersion of NA in the $i \mathrm{PP}$ melt, it is not part of the scope of this work and will be reported separately.

Effect of OXA3,6 on iPP Crystallization after Shear. In the previous section we observed that the introduction of various concentrations of OXA3,6 in iPP lowers the (shear) viscosity compared to pure $i \mathrm{PP}$. This discrepancy in shear response has implications for the processing of the materials since the nucleated sample is less susceptible to the generation and crystallization of shear-induced precursors during flow, as is evident from the slit flow data depicted in Figure 5. To evaluate this behavior and the effect of the nucleating agent on the crystallization morphology, synchrotron radiation studies (SAXS) were performed to monitor the crystallization of $i \mathrm{PP}$ after the application of shear. The shear protocols used are described in the Experimental Section (Figure 1). Figure 10 depicts the characteristic 2D-SAXS patterns obtained for nonsheared $\left(\dot{\gamma}=0 \mathrm{~s}^{-1}\right)$ and several sheared samples $(\dot{\gamma}=10$, 40 , and $200 \mathrm{~s}^{-1}$ ) after cooling to $100{ }^{\circ} \mathrm{C}$. In general, for the samples crystallized under quiescent conditions we observe the presence of an isotropic halo in scattering intensity, corresponding to the long period $\left(L_{\mathrm{p}}\right)$ of crystal lamella of isotropically crystallized $\alpha-i \mathrm{PP}$. The application of a shear pulse at $170{ }^{\circ} \mathrm{C}$ results in the appearance of two vertical lobes in the 2D-SAXS pattern upon cooling, on top of the previously observed isotropic halo. These lobes in scattering intensity are characteristic for transcrystallization or shish-kebab morphology and correspond to the long period $\left(L_{\mathrm{p}}\right)$ of crystal kebabs. Please note that the peak in the equator, corresponding to the presence of shishes formed during shear, is not apparent after cooling due to the high scattering intensity of the crystalline component. Images depicting the equatorial streak directly after the application of shear pulse conducted under various wall-shear rates are provided in Figure S10. In general, from Figure $\mathrm{S} 10$, we can observe that the $\mathrm{PP} / \mathrm{OXA3,6}$ system forms a less intense shish signal than the pure $i \mathrm{PP}$ sample upon the application of the same shear rates. These finding are in line with the observation that the $i \mathrm{PP} / \mathrm{OXA3,6}$ system exhibits a lowered viscosity than the pure $i \mathrm{PP}$ sample. Furthermore, the decrease in shish intensity in the presence of OXA3,6 indicates that OXA3,6 particles do not induce particle-assisted shish 
formation, at least none that can be detected in the length scales probed by SAXS. ${ }^{15}$

Upon integration of the 2D-SAXS patterns in the meridional direction (Figure 11), information can be obtained on the $L_{\mathrm{p}}$

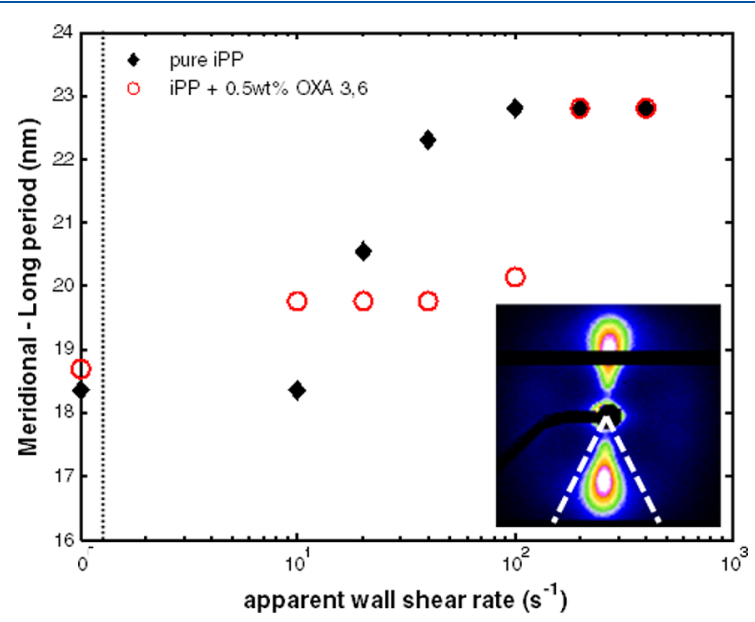

Figure 11. Long period $\left(L_{\mathrm{p}}\right)$ as a function of apparent wall shear rate $(\dot{\gamma})$, as obtained from the peak maximum in meridional scattering intensity of the 2D SAXS patterns.

of the crystal kebabs. Please note that since the MPR bar is cooled from the outside, the sample experiences a gradient in temperature during cooling, resulting in different cooling and crystallization rates throughout the sample. As a result, both the lamellar thickness and crystallinity can vary throughout the sample, thus making the $L_{\mathrm{p}}$ observed in SAXS experiments only suitable for qualitative analysis of the crystallization process. ${ }^{53}$ That being said, for both pure $i \mathrm{PP}$ and $i \mathrm{PP}$ blended with OXA3,6 we observe that after quiescent crystallization a meridional $L_{\mathrm{p}}$ of roughly $18.5 \mathrm{~nm}$ of the isotropic crystallites is found. The application of a shear pulse results, as expected, in the formation and crystallization of shear-induced precursors that facilitate kebab growth upon cooling, thereby increasing the $L_{\mathrm{p}}$ in the meridional scattering direction. ${ }^{54}$ For example, for pure $i \mathrm{PP}$ an upward shift in $L_{\mathrm{p}}$ from 18.3 to $22.8 \mathrm{~nm}$ is observed as the fraction of kebabs increase. This kebab growth, starting already at $\dot{\gamma}>10 \mathrm{~s}^{-1}$, is in good agreement with the earlier observed formation and crystallization of shear-induced precursors for the pure iPP (Figure 5A).

Interestingly, iPP blended with OXA3,6 shows a different trend in the creation of the shish-kebab morphology compared to the pure iPP sample. To recall, in the presence of OXA3,6 the application of $10 \geq \dot{\gamma}<100 \mathrm{~s}^{-1}$ generates weak lobes in the meridional scattering intensity, combined with a minor increase in $L_{\mathrm{p}}$ from 18.7 to $19.8 \mathrm{~nm}$. This indicates that in the presence of OXA3,6 the application of $\dot{\gamma}=10 \mathrm{~s}^{-1}$ is already sufficient to create kebab growth upon cooling. However, as is deduced from Figure 5B, at these low shear rates, no shearinduced precursor formation and crystallization is expected.
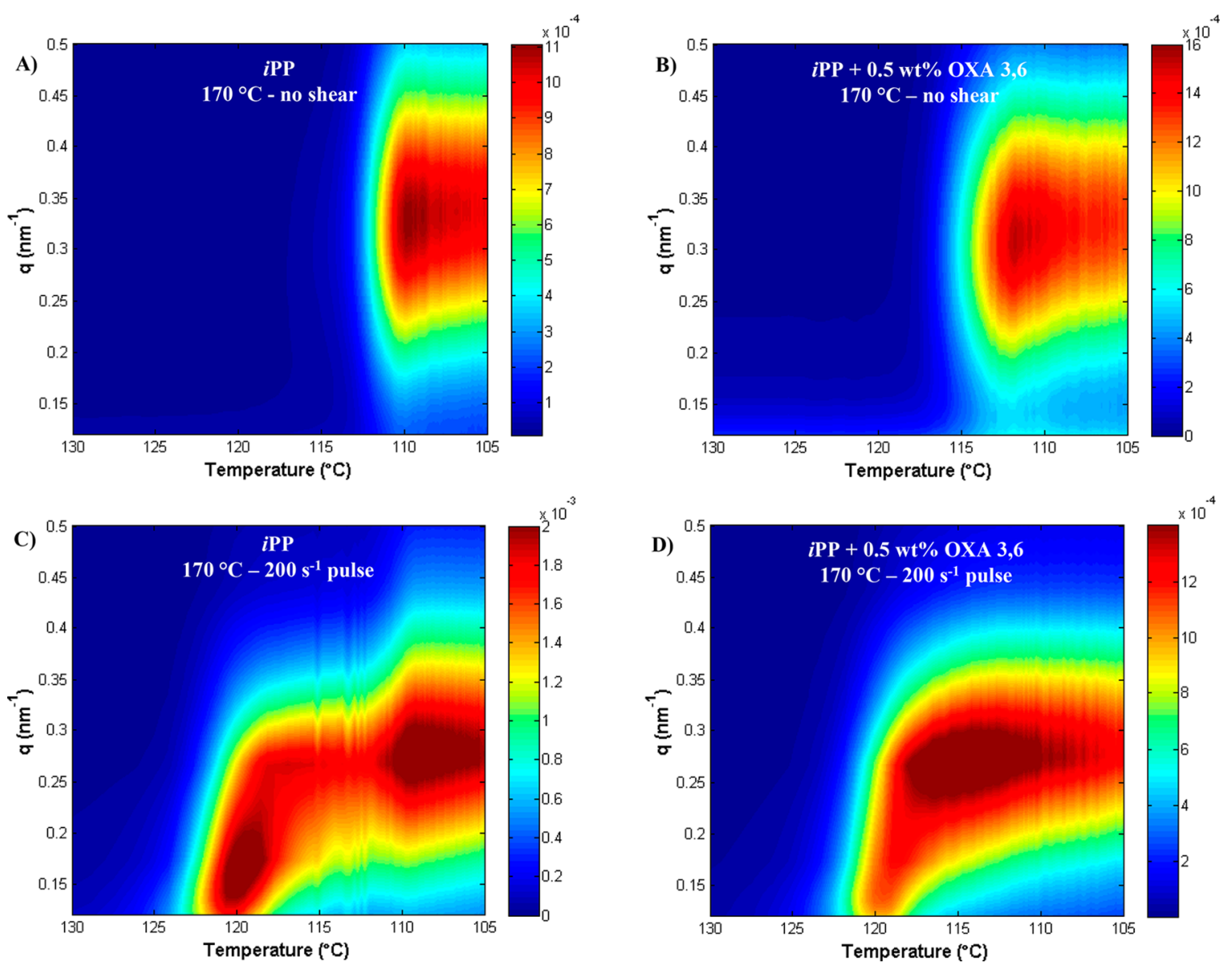

Figure 12. Changes in 1D SAXS patterns observed over time during cooling in the temperature range between 140 and $105{ }^{\circ} \mathrm{C}$ without the application of shear (A, B) or after the application of a shear pulse at $\dot{\gamma}=200 \mathrm{~s}^{-1}$ (C, D) for both non-nucleated iPP (A, C) and iPP containing 0.5 wt \% OXA3,6 (B, D). 

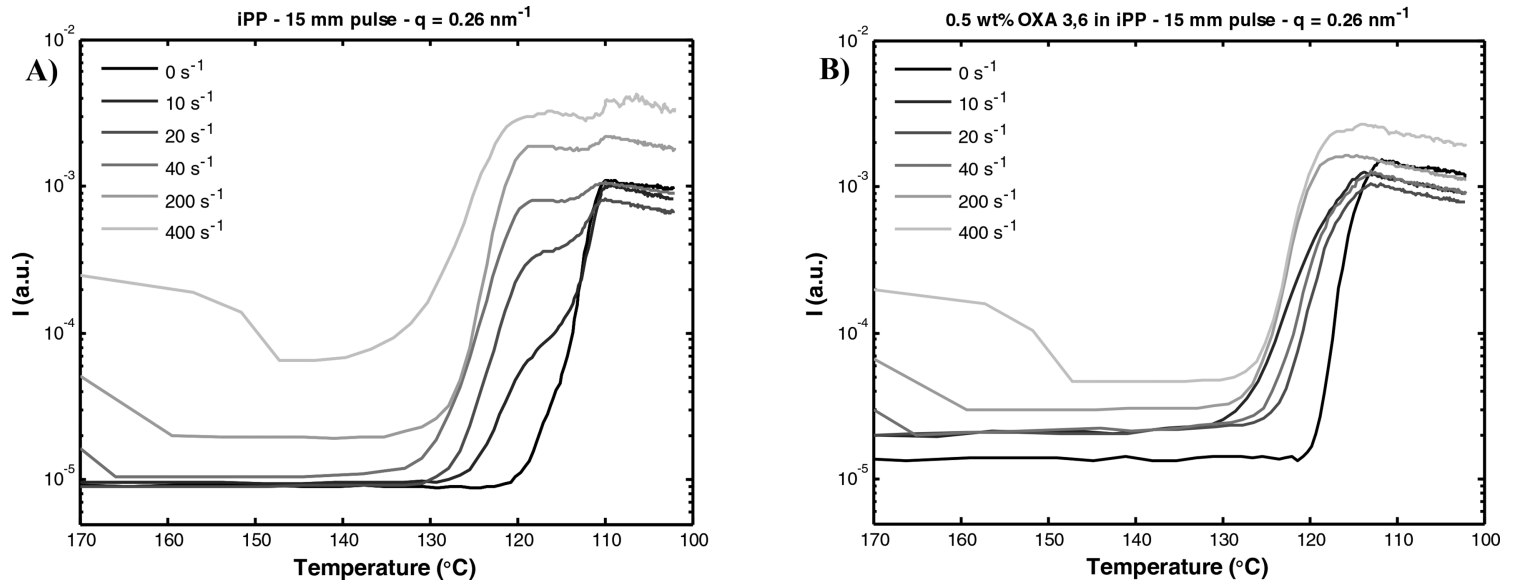

Figure 13. Evolution in in scattering intensity at $q=0.26 \mathrm{~nm}^{-1}$ observed during cooling for (A) pure iPP and (B) $i$ PP blended with OXA3,6 after the application a shear pulse of $15 \mathrm{~mm}$ at various wall shear rates.

Retrospectively, no meridional scattering signal in SAXS intensity is detected in Figure $S 10$ for $i \mathrm{PP}$ blended with OXA3,6 at this shear rate, confirming that indeed no (particleassisted) shish formation ${ }^{15}$ occurs at the shear rate of $10 \mathrm{~s}^{-1}$. Additionally, the absence of the equatorial SAXS peak at $\dot{\gamma}=10$ $\mathrm{s}^{-1}$ indicates that the OXA3,6 particles themselves are too widely spaced and/or too big to provide the desired electron density fluctuation required for the detection of an equatorial SAXS signal. This is in agreement with the observations from optical microscopy depicted in Figure 4. It has been earlier reported by us that the OXA3,6 nucleating agent crystals provide a surface for nucleation, which on shear alignment will provide the required orientation facilitating the transcrystallization morphology. ${ }^{4}$ For this reason, we attribute the kebab crystal growth observed after the application of low $\dot{\gamma}$ to the shear-alignment of the OXA3,6 crystals. A further increase in the $L_{\mathrm{p}}$ to $22.7 \mathrm{~nm}$ is observed only after the application of a shear pulse with $\dot{\gamma} \geq 100 \mathrm{~s}^{-1}$, indicating the presence of a shear-induced shish-kebab morphology. These findings are again in good agreement with the onset of the formation of shear-induced precursors and their crystallization (Figure 5B). Overall, this data indicates that the crystallization morphology generated upon cooling of sheared $i \mathrm{PP}$ in the presence of OXA3,6 is the combined contribution of kebab growth on (1) the shear-aligned nucleating agent and (2) the shear-induced $i$ PP shishes.

To obtain more insight into the role of OXA3,6 on the crystallization process of $i \mathrm{PP}$, the changes in SAXS scattering intensity were monitored online during cooling. Figure 12 depicts examples of the development of the 1D-SAXS patterns during crystallization for both systems after the application of a shear pulse of $\dot{\gamma}=0 \mathrm{~s}^{-1}$ (quiescent crystallization) and $\dot{\gamma}=200$ $\mathrm{s}^{-1}$. For the quiescently cooled samples (Figures 12A,B) we can clearly observe that the presence of OXA3,6 enhances the crystallization process as the rise in scattering intensity, corresponding to the onset of crystallization, is observed at $115^{\circ} \mathrm{C}$. For comparison, the onset of crystallization in the pure $i$ PP sample is observed at $112^{\circ} \mathrm{C}$. Similarly, kebab growth can be observed for both sheared samples (Figures 12C,D), indicated by the rise of the in scattering intensity around $q=$ $0.18 \mathrm{~nm}^{-1}$ starting from $125{ }^{\circ} \mathrm{C}$. For the sheared pure iPP sample (Figure 12C) we observe that the crystallization proceeds in consecutive steps; in the first step, the onset of crystallization of kebabs starts at $125{ }^{\circ} \mathrm{C}\left(L_{\mathrm{p}}=34 \mathrm{~nm}\right.$ at $q=$
$0.18 \mathrm{~nm}^{-1}$ ) immediately followed by the filling in process, thereby changing the scattering intensity and periodicity in the system and shifting the $L_{\mathrm{p}}$ to $24 \mathrm{~nm}$ (at $q=0.26 \mathrm{~nm}^{-1}$ ). In the second step, crystallization of the isotropic crystallites in the core of the MPR bar is observed, again starting at $112^{\circ} \mathrm{C}\left(L_{\mathrm{p}}=\right.$ $24 \mathrm{~nm}$ at $\left.q=0.26 \mathrm{~nm}^{-1}\right)$. This indicates that the crystallization of the isotropic core is not noticeably affected by the shear protocol and displays an onset of crystallization comparable to that of the quiescently crystallized sample. Such crystallization behavior is characteristic for shear-induced polymer samples, where the outer (shear) layers crystallize first, followed by separate crystallization of the inner layers and the isotropic core. $^{55}$ In contrast, the combined effect of shear in the presence of OXA3,6 significantly enhances crystallization of the isotropic core which occurs almost simultaneously with the kebab growth (Figure 12D). Though this crystallization process is also likely to proceed in two stages as for the pure iPP, the stages are difficult to distinguish.

Crystallization studies after the application of various shear rates were performed to identify the exact role of the apparent wall shear rate on the crystallization process. Figure 13 depicts the evolution of the integrated 1D SAXS intensity signal at $q=$ $0.26 \mathrm{~nm}^{-1}$ during the crystallization process. We deliberately chose to monitor the change in scattering intensity at $q=0.26$ $\mathrm{nm}^{-1}$ since this signal contains both the contributions from the kebabs growing close to the wall and the isotropic crystallites growing in the core of the MPR bar, as is visible from Figure 12.

From Figure $13 \mathrm{~A}$ it is apparent that for pure iPP the characteristic two-step crystallization behavior is found for all shear rates. Despite the increasing contribution in scattering intensity from the kebab growth, the crystallization of the isotropic core is detected at $112{ }^{\circ} \mathrm{C}$ for the investigated shear rates. Similar to the earlier observations, crystallization of $i \mathrm{PP}$ blended with OXA3,6 after shear results into a rapid increase in scattering intensity where we are unable to distinguish between kebab growth and isotropic crystal growth. In addition, we observe that an increase in $\dot{\gamma}$ also increases the crystallization rate of the bulk sample while cooling. For example, for $\dot{\gamma} \leq 200 \mathrm{~s}^{-1}$, crystallization of $i$ PP blended with OXA3,6 is completed as the temperature approaches $120^{\circ} \mathrm{C}$ (Figure 13B). In contrast, crystallization of the same sample in the absence of shear or after the application of a shear pulse with $\dot{\gamma}=20 \mathrm{~s}^{-1}$ is only complete below 112 and $115^{\circ} \mathrm{C}$, 
respectively. These findings suggest that the underlying mechanism for the synergistic effect of shear in the presence of OXA3,6 on the crystallization of $i \mathrm{PP}$ is resulting from a modification of the local environment close to the OXA3,6 particles induced by flow. Possible mechanisms include (1) the stabilization of $i \mathrm{PP}$ segments absorbed onto the OXA3,6 particles into their extended chain conformation, (2) increased strain and strain rates of the $i \mathrm{PP}$ matrix near the particles, and (3) extension and stretching of the $i \mathrm{PP}$ chains that contain absorbed segments on the OXA3,6 particles. ${ }^{15}$ Unfortunately, as the OXA3,6 particles and any particle-induced shish formed close to OXA3,6 surface do not provide the required electron density contrast for detection in SAXS, the current set of experiments do not allow further identification of this mechanism. That being said, from the data it is clear that the previously observed shear alignment of the NA aggregates in combination with the interaction between the OXA3,6 and $i \mathrm{PP}$ chains is responsible for the enhanced nucleation efficiency of the whole sample after the application of shear.

Effect of OXA3,6 on iPP Crystal Morphology after Crystallization. To evaluate the crystal morphology and shear layer thickness after crystallization of the samples, the center of the MPR bars obtained after the application of a shear pulse with $\dot{\gamma}=400 \mathrm{~s}^{-1}$ were cut using cryo-microtomy and analyzed using polarized optical microscopy (Figure 14). As expected,
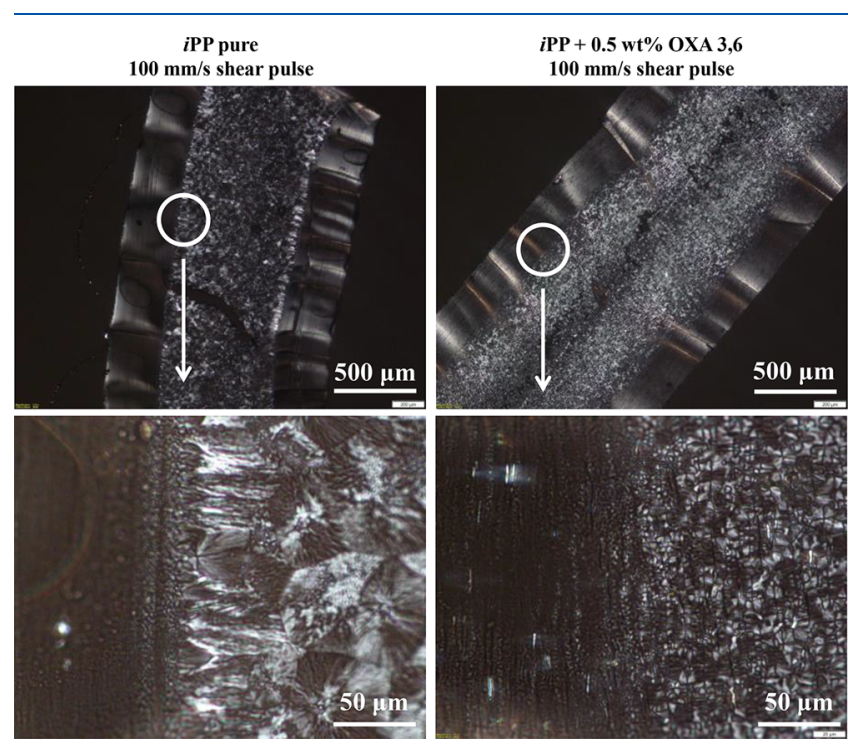

Figure 14. Morphology of slices of the pure $i \mathrm{PP}$ (left) and $i \mathrm{PP}$ containing OXA 3,6 (right) MPR bars crystallized after the application of a shear pulse with $\dot{\gamma}=400 \mathrm{~s}^{-1}$. The bottom figures display a zoom of the interfaces between the shear layer and the isotropic core.

the shear layers in the $i$ PP blended with OXA3,6 $(280 \mu \mathrm{m})$ are smaller than those for the pure $i \mathrm{PP}$ sample $(350 \mu \mathrm{m})$. When zooming in on the interface between the various layers of the pure $i \mathrm{PP}$ sample, we observe a characteristic transition (from left to right) from a shear layer to transcrystallization layer and eventually to the isotropically crystallized core. The presence of the transcrystallization layer is the result from the temporal separation of the crystallization of the shear layer and the core, where the crystallized shear layers provide the surface for transcrystallization.

In contrast, for iPP blended with OXA3,6, no clear differentiation can be made between the various layers; instead, a gradual transition from the skin layer to the isotropic core is observed. Furthermore, when comparing the crystal orientation in the expected isotropic core of the sample containing OXA3,6, one may observe that the crystallites are still exhibiting a layered structure (Figure 14, bottom right), indicating that these crystallites are also oriented along the shear direction. We expect that this is a result from the previously observed shear alignment of the OXA3,6 particles, which is facilitated already under low shear rates (Figure 10). In addition, characteristic for heterogeneously nucleated samples, crystallites in the "isotropic core" are significantly smaller than in the pure iPP sample, resulting from the increased number of nuclei. The absence of the transcrystallization layer combined with the presence of layered $i$ PP crystallites confirms that crystallization of the whole sample indeed proceeds more instantaneous due to shear alignment of the OXA3,6 particles, resulting in a more gradual transition in crystal morphology.

\section{CONCLUSIONS}

In this study we have evaluated the effect of the oxalamide based nucleating agent OXA3,6 on the melt and crystallization behavior of $i$ PP. Despite the fact that both non-nucleated and nucleated samples are comparable in molecular weight according to GPC analysis, we observe a significant suppression in viscosity for $i \mathrm{PP}$ containing 0.5 wt \% OXA3,6. Both from MPR and rheology experiments this suppression is observed, and this behavior could not be explained using molecular modeling. We hypothesize that the viscosity suppression in the presence of OXA3,6 is a result from physical absorption of $i \mathrm{PP}$ chains on the nucleating agent surface, thereby excluding high molecular weight $\left(M_{\mathrm{w}}\right)$ chains of $i$ PP from the melt. This proposed mechanism for the suppression of viscosity appears similar to that encountered in the presence of relatively rigid nanoparticles such as CNTs, graphene, or silica. However, the difference is that the selfassembly of OXA3,6 at high temperature provides the flexibility of the aggregates to adopt the desired conformations required for the nucleation of $i \mathrm{PP}$ upon cooling, especially after the application of shear. The application of shear in the presence of OXA3,6 results in the shear alignment of the NA particles already at low shear rates, thereby providing a surface for crystal kebab growth and enhancing the nucleation and crystallization upon cooling. This behavior proceeds in addition to the enhancement in crystallization through shearinduced crystallization close to the wall. As a result, the crystallization of the whole MPR bar, hence both the shear layers and the core, proceeds almost simultaneously. Because of this simultaneous crystallization, the traditional layered morphology, especially the transcrystalline layer, is absent. Instead, the expected transcrystalline layer and the isotropic core are replaced by multiple layers of crystallites aligned along the shear direction. This combined suppression in melt viscosity and enhanced nucleating efficiency after shear makes the OXA3,6 nucleating agent a highly promising candidate for rapid processing under both low and high shear conditions.

\section{ASSOCIATED CONTENT}

\section{S Supporting Information}

The Supporting Information is available free of charge on the ACS Publications website at DOI: 10.1021/acs.macromol.8b00489. 
Additional information on the tube model and verification of the used parameters, usage of the tube model to examine the effect of long chain branching (LCB) (Figures S1-S7), melt behavior of iPP blended with OXA3,6 in various concentrations (Figure S8), melt behavior of $i \mathrm{PP}$ at various temperatures (Figure S9), and effect of wall-shear rate on shish scattering (Figure S10) (PDF)

\section{AUTHOR INFORMATION}

\section{Corresponding Author}

*E-mail karel.wilsens@maastrichtuniversity.nl (C.H.R.M.W.). ORCID $\odot$

Carolus H. R. M. Wilsens: 0000-0003-3063-9510

Gerrit W. M. Peters: 0000-0001-7208-5128

Sanjay Rastogi: 0000-0002-7804-7349

Notes

The authors declare no competing financial interest.

\section{ACKNOWLEDGMENTS}

NWO (Nederlandse Organisatie voor Wetenschappelijk Onderzoek) is acknowledged for providing beam-time at the ESRF. The DUBBLE (Dutch Belgian beamline) staff is acknowledged for supporting the X-ray experiments.

\section{REFERENCES}

(1) Blomenhofer, M.; Ganzleben, S.; Hanft, D.; Schmidt, H.-W.; Kristiansen, M.; Smith, P.; Stoll, K.; Mäder, D.; Hoffmann, K. "Designer" Nucleating Agents for Polypropylene. Macromolecules 2005, 38, 3688.

(2) Varga, J.; Menyhárd, A. Effect of solubility and nucleating duality of $\mathrm{N}, \mathrm{N}^{\prime}$-dicyclohexyl-2,6-naphthalenedicarboxamide on the supermolecular structure of isotactic polypropylene. Macromolecules 2007, 40, 2422.

(3) Shi, Y.; Xin, Z. Study on variable nucleation efficiency of $\mathrm{N}, \mathrm{N}^{\prime}$ Dicyclohexyl-2,6-naphthalenedicarboxamide on isotactic polypropylene. J. Thermoplast. Compos. Mater. 2016, 29, 1667.

(4) Deshmukh, Y. S.; Wilsens, C. H. R. M.; Leoné, N.; Portale, G.; Harings, J. A. W.; Rastogi, S. Melt-Miscible Oxalamide Based Nucleating Agent and Their Nucleation Efficiency in Isotactic Polypropylene. Ind. Eng. Chem. Res. 2016, 55, 11756-11766.

(5) Ma, P.; Xu, Y.; Wang, D.; Dong, W.; Chen, W. Rapid Crystallization of Poly(lactic acid) by Using Tailor-Made Oxalamide Derivatives as Novel Soluble-Type Nucleating Agents. Ind. Eng. Chem. Res. 2014, 53, 12888.

(6) Shen, T.; Xu, Y.; Cai, X.; Ma, P.; Dong, W.; Chen, M. Enhanced crystallization kinetics of poly(lactide) with oxalamide compounds as nucleators: effect of spacer length between the oxalamide moieties. RSC Adv. 2016, 6, 48365.

(7) Ma, P.; Deshmukh, Y. S.; Wilsens, C. H. R. M.; Hansen, M. R.; Graf, R.; Rastogi, S. Self-assembling process of Oxalamide compounds and their nucleation efficiency in bio-degradable Poly(hydroxyalkanoate)s. Sci. Rep. 2015, 5, 13280.

(8) Deshmukh, Y. S.; Ma, P.; Wilsens, C. H. R. M.; Rastogi, S. Nucleating agents for biopolymers, WO 2013120793 A1, 2013.

(9) Yang, Y.; Liang, R.; Chen, Y.; Zhang, C.; Zhang, R.; Wang, X.; Kong, R.; Chen, Q. Using a Self-Assemblable Nucleating Agent To Tailor Crystallization Behavior, Crystal Morphology, Polymorphic Crystalline Structure, and Biodegradability of Poly(1,4-butylene adipate). Ind. Eng. Chem. Res. 2017, 56 (28), 7910-7919.

(10) Xu, P.; Feng, Y.; Ma, P.; Chen, Y.; Dong, W.; Chen, M. Crystallization behaviours of bacterially synthesized poly(hydroxyalkanoate)s in the presence of oxalamide compounds with different configuration. Int. J. Biol. Macromol. 2017, 104, 624-630.
(11) Ma, P.; Xu, Y.; Wang, D.; Dong, W.; Chen, M. Rapid Crystallization of Poly(lactic acid) by Using Tailor-Made Oxalamide Derivatives as Novel Soluble-Type Nucleating Agents. Ind. Eng. Chem. Res. 2014, 53, 12888-12892.

(12) Xing, Q.; Li, R.; Dong, X.; Luo, F.; Kuang, X.; Wang, D.; Zhang, L. Enhanced Crystallization Rate of Poly(L -lactide) Mediated by a Hydrazide Compound: Nucleating Mechanism Study. Macromol. Chem. Phys. 2015, 216 (10), 1134-1145.

(13) Marco, C.; Ellis, G.; Gómez, M. A.; Arribas, J. M. Comparative study of the nucleation activity of third-generation sorbitol-based nucleating agents for isotactic polypropylene. J. Appl. Polym. Sci. 2002, $84,2440$.

(14) Balzano, L.; Portale, G.; Peters, G. W. M.; Rastogi, S. Thermoreversible DMDBS Phase Separation in iPP: The Effects of Flow on the Morphology. Macromolecules 2008, 41, 5350.

(15) Phillips, A. W.; Bhatia, A.; Zhu, P.-W.; Edward, G. Shish Formation and Relaxation in Sheared Isotactic Polypropylene Containing Nucleating Particles. Macromolecules 2011, 44, 35173528.

(16) Somani, R. H.; Yang, L.; Hsiao, B. S.; Agarwal, P. K.; Fruitwala, H. A.; Tsou, A. H. Shear-Induced Precursor Structures in Isotactic Polypropylene Melt by in-Situ Rheo-SAXS and Rheo-WAXD Studies. Macromolecules 2002, 35, 9096-9104.

(17) Li, L. B.; de Jeu, W. H. Shear-Induced Crystallization of Poly(butylene terephthalate): A Real-Time Small-Angle X-ray Scattering Study. Macromolecules 2004, 37, 5646-5652.

(18) Kumaraswamy, F.; Issaian, A. M.; Kornfield, J. A. ShearEnhanced Crystallization in Isotactic Polypropylene. 1. Correspondence between in Situ Rheo-Optics and ex Situ Structure Determination. Macromolecules 1999, 32, 7537-7547.

(19) Yalcin, B.; Valladares, D.; Cakmak, M. Amplification effect of platelet type nanoparticles on the orientation behavior of injection molded nylon 6 composites. Polymer 2003, 44, 6913-6925.

(20) Jain, S.; Goossens, J. P. G.; Peters, G. W. M.; van Duin, M.; Lemstra, P. J. Strong decrease in viscosity of nanoparticle-filled polymer melts through selective adsorption. Soft Matter 2008, 4, $1848-1854$.

(21) Troisi, E. M.; Portale, G.; Ma, Z.; van Drongelen, M.; HermidaMerino, D.; Peters, G. W. M. Unusual Melting Behavior in Flow Induced Crystallization of LLDPE: Effect of Pressure. Macromolecules 2015, 48 (8), 2551-2560.

(22) Das, C.; Inkson, N. J.; Read, D. J.; Kelmanson, M. A.; McLeish, T. C. B. Computational linear rheology of general branch-on-branch polymers. J. Rheol. 2006, 50 (2), 207-234.

(23) Doi, M.; Edwards, S. F. The Theory of Polymer Dynamics, 1st ed.; International series of monographs on physics; Oxford University Press: Oxford, 1986.

(24) McLeish, T. C. B. Tube theory of entangled polymer dynamics. Adv. Phys. 2002, 51 (6), 1379-1527.

(25) Watanabe, H. Viscoelasticity and dynamics of entangled polymers. Prog. Polym. Sci. 1999, 24 (9), 1253-1403.

(26) Hamad, F. G.; Colby, R. H.; Milner, S. T. Onset of FlowInduced Crystallization Kinetics of Highly Isotactic Polypropylene. Macromolecules 2015, 48 (11), 3725-3738.

(27) Eckstein, A.; Suhm, J.; Friedrich, C.; Maier, R. D.; Sassmannshausen, J.; Bochmann, M.; Mülhaupt, R. Determination of Plateau Moduli and Entanglement Molecular Weights of Isotactic, Syndiotactic, and Atactic Polypropylenes Synthesized with Metallocene Catalysts. Macromolecules 1998, 31 (4), 1335-1340.

(28) Bras, W.; Dolbnya, I. P.; Detollenaere, D.; van Tol, R.; Malfois, M.; Greaves, G. N.; Ryan, A. J.; Heeley, E. Recent experiments on a small-angle/wide-angle X-ray scattering beam line at the ESRF. J. Appl. Crystallogr. 2003, 36, 791.

(29) Portale, G.; Cavallo, D.; Alfonso, C.; Hermida-Merino, D.; van Drongelen, M.; Balzano, L.; Peters, G. W. M.; Goossens, J. P. G.; Bras, W. Polymer crystallization studies under processing-relevant conditions at the SAXS/WAXS DUBBLE beamline at the ESRF. J. Appl. Crystallogr. 2013, 46, 1681. 
(30) Blackadder, D. A.; Le Poidevin, G. J. Dissolution of polypropylene in organic solvents: 1. Partial dissolution. Polymer 1976, 17, 387-394.

(31) Ma, P.; Yu, Q.; Shen, T.; Dong, W.; Chen, M. Strong synergetic effect of fibril-like nucleator and shear flow on the melt crystallization of poly(L-lactide). Eur. Polym. J. 2017, 87, 221-230.

(32) Xu, J.-Z.; Chen, C.; Wang, Y.; Tang, H.; Li, Z.-M.; Hsiao, B. S. Graphene Nanosheets and Shear Flow Induced Crystallization in Isotactic Polypropylene Nanocomposites. Macromolecules 2011, 44, $2808-2818$

(33) Nam, Y.; Shim, K. B.; Kim, S. H.; Kim, B. C. Effect of Silica Nanoparticle on the Quiescent and the Shear Induced Crystallization Behaviors of Poly(trimethylene terephthalate). Polym. J. 2004, 36 (7), 519-530.

(34) D’Haese, M.; van Puyvelde, P.; Langouche, F. Effect of Particles on the Flow-Induced Crystallization of Polypropylene at Processing Speeds. Macromolecules 2010, 43, 2933-294.

(35) D’Haese, M.; Langouche, F.; van Puyvelde, P. On the Effect of Particle Size, Shape, Concentration, and Aggregation on the FlowInduced Crystallization of Polymers. Macromolecules 2013, 46, 34253434.

(36) Patil, N.; Balzano, L.; Portale, G.; Rastogi, S. A Study on the Chain-Particle Interaction and Aspect Ratio of Nanoparticles on Structure Development of a Linear Polymer. Macromolecules 2010, 43, 6749-6759.

(37) Han, R.; Nie, M.; Wang, Q.; Yan, S. Self-Assembly $\beta$ Nucleating Agent Induced Polymorphic Transition from $\alpha$-Form Shish Kebab to $\beta$-Form Highly Ordered Lamella under Intense Shear Field. Ind. Eng. Chem. Res. 2017, 56 (10), 2764-2772.

(38) Ma, Z.; Balzano, L.; Van Erp, T.; Portale, G.; Peters, G. W. M. Short-Term Flow Induced Crystallization in Isotactic Polypropylene: How Short Is Short? Macromolecules 2013, 46, 9249-9258.

(39) Cox, P. W.; Merz, E. H. Correlation of dynamic and steady flow viscosities. J. Polym. Sci. 1958, 28, 619-622.

(40) vander Linden, C.; van Leemput, R. Adsorption Studies of Polystyrene on Silica. J. Colloid Interface Sci. 1978, 67, 63-69.

(41) Felter, R. E.; Ray, L. N. Polymer Adsorption Studies at the Solid-Liquid Interface Using Gel Permeation Chromatography I. Molecular Weight Distribution Along the Adsorption Isotherm. J. Colloid Interface Sci. 1970, 32 (2), 349-360.

(42) Zhang, Q. D.; Lippits, D.; Rastogi, S. Dispersion and rheological aspects of SWNTs in intractable polymers. Macromolecules 2006, 39 (2), 658-666.

(43) Yuryev, Y.; Wood-Adams, P. Rheological properties of crystallizing polylactide: Detection of induction time and modeling the evolving structure and properties. J. Polym. Sci., Part B: Polym. Phys. 2010, 48, 812-822.

(44) Tierney, N. K.; Register, R. A. Synthesis and Melt Dynamics of Model Sulfonated Ionomers. Macromolecules 2003, 36 (4), 11701177.

(45) Chen, Q.; Zhang, Z.; Colby, R. H. Viscoelasticity of entangled random polystyrene ionomers. J. Rheol. 2016, 60, 1031-1040.

(46) Hawke, L. G. D.; Ahmadi, M.; Goldansaz, H.; van Ruymbeke, E. Viscoelastic properties of linear associating poly(n-butyl acrylate) chains. J. Rheol. 2016, 60, 297-310.

(47) Ramakrishnan, V. Universal Viscosity Behavior of Polycondensation Polymer/Silica nanocomposites. PhD thesis Eindhoven University of Technology, the Netherlands, 2016.

(48) Liu, K.; Ronca, R.; Andablo-Reyes, E.; Forte, G.; Rastogi, S. Unique Rheological Response of Ultrahigh Molecular Weight Polyethylenes in the Presence of Reduced Graphene Oxide. Macromolecules 2015, 48 (1), 131-139.

(49) Zhang, Q. D.; Lippits, D.; Rastogi, S. Dispersion and rheological aspects of SWNTs in intractable polymers. Macromolecules 2006, 39 (2), 658-666.

(50) Shchetnikava, V.; Slot, J. J. M.; van Ruymbeke, E. A Comparison of Tube Model Predictions of the Linear Viscoelastic Behavior of Symmetric Star Polymer Melts. Macromolecules 2014, 47 (10), 3350-3361.
(51) Milner, S. T.; McLeish, T. C. B.; Young, R. N.; Hakiki, A.; Johnson, J. M. Dynamic dilution, constraint release, and star-linear blends. Macromolecules 1998, 31 (26), 9345-9353.

(52) Likhtman, A. E.; McLeish, T. C. B. Quantitative theory for linear dynamics of linear entangled polymers. Macromolecules 2002, 35 (16), 6332-6343.

(53) Caelers, H. J. M.; Govaert, L. E.; Peters, G. W. M. The prediction of mechanical performance of isotactic polypropylene on the basis of processing conditions. Polymer 2016, 83, 116-128.

(54) Roozemond, P. C.; van Drongelen, M.; Ma, Z.; Spoelstra, A. B.; Hermida-Merino, D.; Peters, G. W. M. Self-regulation in flow induced structure formation of polypropylene. Macromol. Rapid Commun. 2015, 36, 385-390.

(55) Schrauwen, B. A. G.; v. Breemen, L. C. A.; Spoelstra, A. B.; Govaert, L. E.; Peters, G. W. M.; Meijer, H. E. H. Structure, deformation, and failure of flow-oriented semicrystalline polymers. Macromolecules 2004, 37, 8618-8633. 